\title{
Piloted Simulator Evaluation Results of Flight Physics Based Stall Recovery Guidance
}

\author{
Thomas Lombaerts* Stefan Schuet ${ }^{\dagger}$ Vahram Stepanyan ${ }^{\ddagger}$ John Kaneshige $^{\S}$ \\ Gordon Hardy "Kimberlee Shish" and Peter Robinson** \\ NASA Ames Research Center, Moffett Field, CA 94035
}

\begin{abstract}
Recent studies have shown that loss of control in flight is the most frequent primary cause of accidents. A significant share of accidents in this category can be remedied by upset prevention if possible, and by upset recovery if necessary, in this order of priorities. One of the most important upsets to recover from is stall. Recent accidents have shown that a correct stall recovery maneuver remains a big challenge in civil aviation, partly due to a lack of pilot training. A possible strategy to support the flight crew in this demanding context is calculating a recovery guidance signal, and showing this signal in an intuitive way on one of the cockpit displays, for example by means of the flight director. Different methods for calculating the recovery signal, one based on fast model predictive control and another using an energy based approach, have been evaluated in four relevant operational scenarios by experienced commercial as well as test pilots in the Vertical Motion Simulator at NASA Ames Research Center. Evaluation results show that this approach supports the pilots in improving their stall recovery performance.
\end{abstract}

\section{Introduction}

RECENT aircraft accident statistics show that loss of control in flight is the primary category of accidents R that occurs most frequently ${ }_{\text {th }}^{[13}$ A significant number of these accidents were related to stall, such as

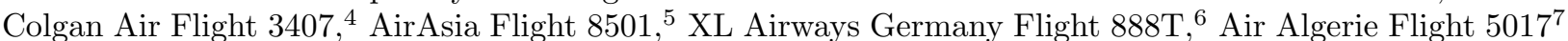

\footnotetext{
*Senior Aerospace Research Engineer, Stinger Ghaffarian Technologies, Intelligent Systems Division, Mail Stop 269-1, AIAA Associate Fellow, thomas.lombaerts@nasa.gov

$\dagger$ Computer Engineer, Intelligent Systems Division, Mail Stop 269-3, AIAA member.

¥Senior Research Scientist, Universities Space Research Association, Intelligent Systems Division, Mail Stop 269-1, AIAA Senior Member.

$\S$ Computer Engineer, Intelligent Systems Division, Mail Stop 269-1, AIAA member.

『Research Pilot, Science Applications International Corporation, Moffett Field, California 94035, AIAA member.

" Aerospace Engineer, Intelligent Systems Division, Mail Stop 269-1, AIAA member.

**Computer Scientist, Fault Protection Systems, Intelligent Systems Division, Mail Stop 269-2, AIAA Member.
} 


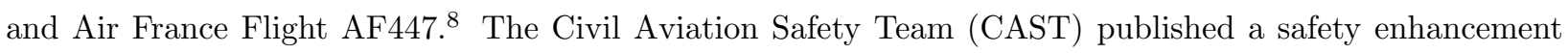
plan comprising a list of recommendations that include research to develop and implement technologies for effective upset prevention and recovery training. 9 In large transport aircraft, an unusual attitude is usually defined as a nose up pitch attitude greater than 25 degs, a nose down pitch attitude greater than 10 degs, a bank angle greater than 45 degs or flight within these parameters but with airspeeds inappropriate for the conditions $[9]$ The following list of priorities can be found in the CAST recommendations:

1. The first priority is to ensure that these parameters are never exceeded (upset prevention).

2. The second priority is to correct any exceedence as rapidly as possible (upset recovery).

3. The third is to understand the principles involved in recovering from an 'upset'.

4. The fourth priority is never to stall the aircraft. A fully stalled aircraft is out of control - recovery from the stall must be achieved before initiating recovery from any unusual attitude (stall recovery before any other upset recovery).

\section{A. Research Goals}

Previous research of the authors, addressing the CAST recommendations for research, development, and implementation of technologies for enhancing flight crew awareness of airplane energy state (SE 207) ${ }^{10}$ has focused on upset prevention $\frac{11}{14}$ by means of safe flight envelope estimation and protection. It is important to understand that certain system failures can degrade these protections such that the aircraft is left with reduced or no protections and become, in effect, a 'basic' aircraft. The next logical step is upset recovery, which is needed in case upset prevention would not be successful (also due to for example atmospheric disturbances, malfunctions or damage). In the category of upset recovery, stall recovery should be prioritized. In SE 207, the following research area is mentioned: 'Develop and refine algorithms and display strategies to provide control guidance for recovery from approach-to-stall or stall.' Recovery techniques for stall should specifically take the following into account:

1. not exceeding the structural limits of the aircraft and maintaining some degree of passenger comfort: this can be done by limiting the load factor $n_{z}$;

2. not bringing the aircraft into a secondary stall, which is caused by attempting to hasten the completion of a stall recovery before the aircraft has regained sufficient flying speed: this is to be achieved by ensuring that $\alpha$ does not exceed $\alpha_{\text {stall }}$ during the recovery maneuver;

3. minimizing the altitude loss during the recovery maneuver, which is especially important for stalls at lower altitudes;

4. avoiding loss of controllability when the stabilizer is deflected in an extreme position. When full thrust is required in a stall recovery maneuver, it is possible that the elevators cannot generate enough countermoment to eliminate the combined pitching behavior caused by the stabilizer and thrust provided by engines mounted under the wings. For this purpose, maximum thrust should be limited based on the total pitching moment which can still be compensated for by the elevators.

The Federal Aviation Authority has issued an Advisory Circular on Stall Prevention and Recovery Training. Although the procedure should apply to the majority of today's airplanes, manufacturer-recommended procedures may deviate from those included in this AC due to specific airplane characteristics. A summary of the FAA Stall Recovery Template ${ }^{15}$ can be found in Table 1 and a sequence of events for stall recovery is shown in Fig. 1. 
Table 1. Summary of FAA Stall Recovery Template, modified from source: 15]

\section{Step 1 Disconnect autopilot and autothrottle/autothrust}

Rationale: Manual control is essential to recovery in all situations. Avoid inadvertent changes by the automation which may not be easily recognized or appropriate, especially in high workload situations. It may be very important in out-of-trim situations to ensure that the pitch attitude does not increase when disconnecting the autopilot.

Step 2 a) Nose down pitch control until stall indications are eliminated

b) Nose down pitch trim as needed

Rationale: a) Reducing the angle of attack is crucial for recovery. This will also address autopilot-induced excessive nose up trim.

b) If the control column does not provide sufficient response, pitch trim may be necessary. However, excessive use of pitch trim may aggravate the condition, or may result in loss of control or high structural loads.

\section{Step 3 Bank wings level}

Rationale: This orients the lift vector upward for recovery.

\section{Step 4 Apply thrust as needed}

Rationale: During a stall recovery, maximum thrust is not always needed. A stall can occur at high thrust or at idle thrust. Therefore, the thrust is to be adjusted accordingly during the recovery. For airplanes with engines installed below the wing, applying maximum thrust may create a strong nose-up pitching moment if airspeed is low.

Step $5 \quad$ Retract speed brakes and spoilers

Rationale: This will improve lift and stall margin.

Step 6 Return to the desired flightpath

Rationale: Apply gentle action for recovery to avoid secondary stalls, return to desired flightpath.

\begin{tabular}{|c|c|c|c|c|}
\hline onset to stall & stall occurrence & accelerating dive & $\begin{array}{l}\text { stall recovery } \\
\text { pitch up }\end{array}$ & out of stall \\
\hline $\begin{array}{l}\text { Decreasing airspeed, } \\
\text { increasing angle of attack }\end{array}$ & 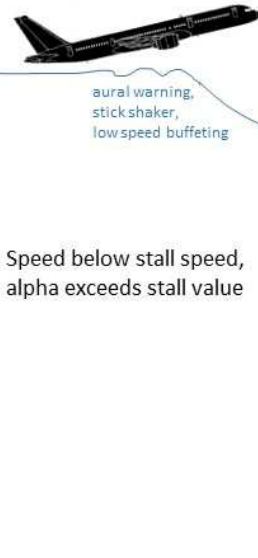 & $\begin{array}{l}\Delta \mathrm{h} \\
\text { Trade altitude for speed, } \\
\text { potential } \rightarrow \text { kinetic energy }\end{array}$ & $\begin{array}{l}\text { Transition to level flight, } \\
\text { avoiding secondary stalls } \\
\text { or overstressing structure } \\
\text { Pitch up but stay below } \\
\text { maximum pitch limit, } \\
\text { avoid high load factors } \\
\text { Trade off between total } \\
\text { altitude loss and safety } \\
\text { buffer for alpha and load. }\end{array}$ & $\begin{array}{l}\text { Establish level flight or } \\
\text { climb } \\
\text { Back to trim condition or } \\
\text { execute go around }\end{array}$ \\
\hline
\end{tabular}

Figure 1. Sequence of events for stall recovery 


\section{B. Literature survey}

Most literature focuses on the more general upset recovery, rather than specific stall recovery. Belcastro gives a comprehensive global overview of LOC/Upset prevention and recovery ${ }^{[16}$ Upsets comprise abnormal attitudes, abnormal trajectories and stall/departures. Gandhi et a ${ }^{17}$ presented a joint human/automated upset recovery system, which is targeted at autonomous upset recovery for UAV's. Recovery strategies were optimized off-line, followed by a small scale pilot in the loop study. Overall findings were: (1.) the time to recover and the amount of altitude lost during recovery were reduced; (2.) the likelihood that the pilot exceeds the structural limits was reduced; and (3.) there was a significant reduction in the pilot workload. The optimization was achieved by means of a constrained nonlinear programming solver. The objective is to return to straight and level flight while minimizing altitude loss, while staying within the structural limits of the vehicle $\left(n_{z}\right)$ and avoiding secondary stalls $(\alpha)$. The recovery guidance signal was presented in the primary flight display PFD as shown in Fig. 2(a). Richards et al discussed the improvement of the aforementioned upset recovery strategy through explicit consideration of distressed pilot dynamics in the generation of recovery sequences, which leads to more desirable responses of the pilot/vehicle system ${ }^{18}$ The pilot model is conventional, consisting of a lead lag filter with an exponential factor for the effective time delay. In a later publication of the same research team, $\frac{19}{19}$ this work is further developed as an in-cockpit cueing system for upset recovery and evaluated in the simulator. This involves a new type of visualization including thrust guidance, as shown in Fig. 2(b) Also force feedback has been included. The following upset conditions have been investigated: high altitude stall, low altitude flaps up stall, low altitude flaps down stall, high bank condition and nose high condition. The performance metrics have been defined as: altitude loss, load factor excursions, angle of attack excursions, final airspeed error and proximity to straight-and-level trim condition. In the latest publication of this research team, Richards et al20 discuss the development and evaluation of a similar approach for upset recovery that is robust to pilot behavior variation and is able to accommodate vehicle failures and impairment. The Damage Adaptive Guidance for piloted Upset Recovery (DAGUR) system provides continuous closed-loop recovery guidance via visual cues to reduce instances of inappropriate pilot reaction and pilot inaction. Adaptation enables the recovery module to provide appropriate guidance even in cases of vehicle damage or impairment. The recovery guidance system is also specifically designed to be robust to variations in pilot dynamic behavior (including behavior associated with high-stress situations). Stepanyan et al ${ }^{21}$ developed stall recovery guidance algorithms based on constrained control approaches. This has been done in two ways, namely an input constrained control approach providing flight path angle or altitude guidance, which is PCH (pseudo control hedging) based, and a state constrained control approach guiding the pitch attitude angle. A more extensive literature review, including other research that was not (yet) tested in a simulator, can be found in Ref. [22].

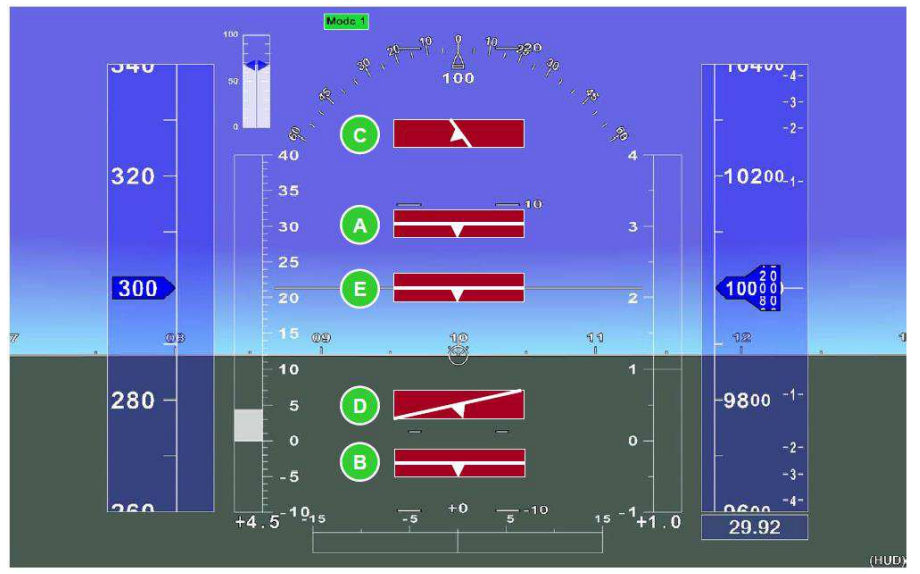

(a) PFD style in 17

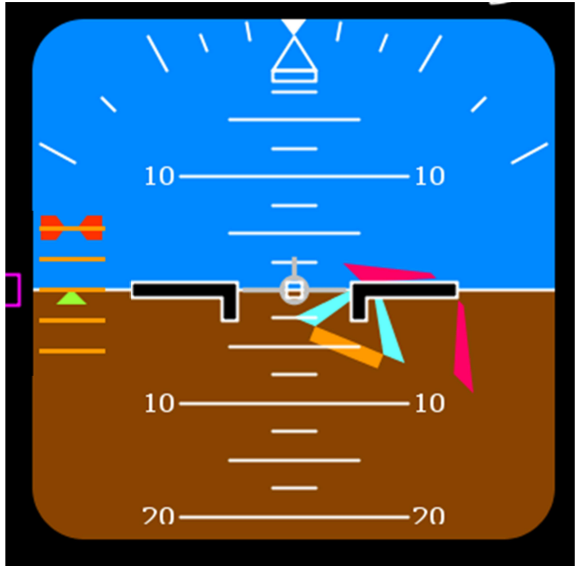

(b) PFD style in 19

Figure 2. Different styles of recover guidance in the PFD as found in the literature 


\section{Paper Focus and Structure}

In the previous phase of this research project, different physics based stall recovery guidance algorithms have been developed. These guidance algorithms are based on energy management, pseudo-control hedging, and model-predictive control (i.e., optimal control). The energy management approach exploits the knowledge of the flight mechanics in a total energy control setup by means of dynamic inversion. ${ }^{22}$ The pseudo-control hedging-based algorithm calculates a pitch angle guidance signal by inverting the aircraft pitch rate dynamics when the angle of attack exceeds the stall limit.21 For the model-predictive control approach, an optimal recovery is computed using a fast convex optimization algorithm, 23 which is capable of running reliably from inside the Ames Vertical Motion Simulator (VMS) frame calculation loop on a $1.25 \mathrm{GHz}$ DEC-Alpha, Real-Time Operating System. This paper describes how a couple of these algorithms have been evaluated by experienced commercial as well as test pilots in the high-fidelity Vertical Motion Simulator (VMS) at NASA Ames Research Center (ARC). The algorithms performance has been investigated across four relevant scenarios, simulating different stall entry conditions. These are a high altitude stall, a low altitude flight condition with bank (with and without excessive nose up trim), and a descent for final approach. All these scenarios were inspired by real life accidents and incidents and will be discussed in more detail later in this paper. This paper serves primarily as a summary of the research project. A full technical report can be found in Ref. 24. It should be noted that there are many stall prevention and awareness features in a nominal aircraft nowadays, from a minimum speed indication on the primary flight display, an aural stall warning, a stick shaker, to low speed buffeting. However, it has been found that these were not successful in preventing the aforementioned air accidents. In this research the emphasis was placed on improving stall recovery. While the aural warning, stick-shaker, and stall buffeting behavior were included in the scenarios for realism, the study participants were asked to ignore them during the initial approach-to-stall.

The structure of this paper is as follows. First the simulation model is described in Sec. III Thereafter, the essentials of the two guidance algorithms are briefly summarized in Sec. III] Sec. IV elaborates in depth on the experiment method, by discussing the technologies (Sec. IVIA), apparatus (Sec. IV]B), participants (Sec. IVIC), measures (Sec. IVID) as well as procedures for all the scenarios (Sec. IV]E). The results are discussed in Sec. V] Finally, Sec. VI presents the conclusions.

\section{Simulation Model}

It is important to use a simulation model with accurate post-stall characteristics, since this research focuses primarily on this flight regime. For this reason, the Generic Transport Model GTM ${ }^{25}$ was used, as shown in Fig. 3. It is a full scale simulation model of a generic aircraft similar to a Boeing 757 , based on a subscale polynomial aerodynamic database, and adapted with Reynolds Number corrections. This simulation model was implemented in the FLTz simulation environment. ${ }^{26}$ Analysis of the steady state aerodynamic data of the simulation model has revealed that deploying flaps (and leading edge slats) increases lift, but has a very small influence on the stall angle of attack, as can be seen in Fig. 4(a). Also the polars are shown in Fig. 4(b).

In order to hold steady level flight at slower airspeed, an aircraft must fly at higher angle of attack. However, as the angle of attack is increased there comes a point where an associated increase in drag requires more thrust to hold the airspeed constant. When more thrust is required to fly slower, the aircraft is said to be on the back side of the power curve. When operating in the backside of the power curve, the speed damping derivative is negative, which implies airspeed cannot be regulated. Induced drag is dominant over viscous drag as a result of the increased lift as airspeed is reduced. Furthermore, in situations at higher altitudes, it is even possible that the thrust required exceeds the maximum thrust available. This means that even with full throttle, the aircraft can decelerate and stall if the pilot attempts to hold level flight. For these reasons, it is important to end up on the front side of the power curve at the end of a high altitude stall recovery. This means recovering with sufficient airspeed so that the aircraft can accelerate without the need for additional altitude loss.

This research considers an unaugmented airplane. All stability and control augmentation functions are switched off except for the yaw damper. 


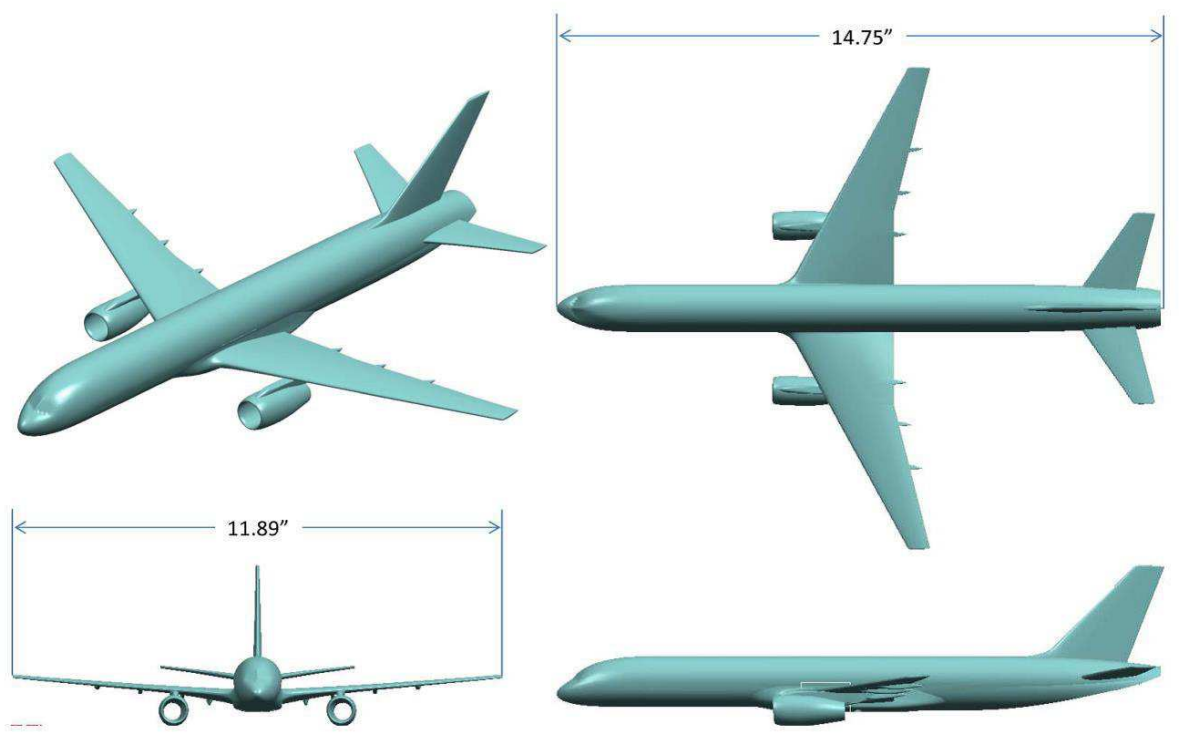

Figure 3. Generic Transport Model GTM with subscale dimensions, source: 27.

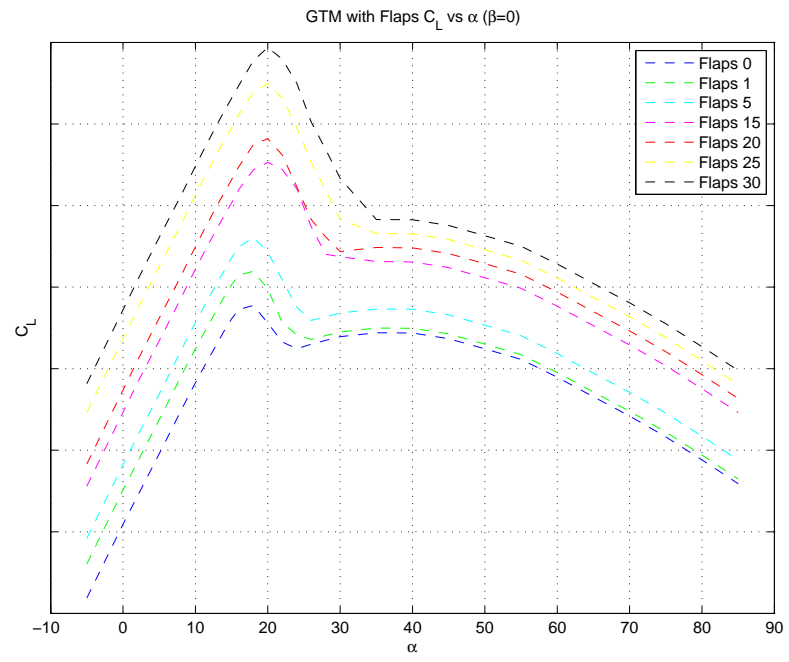

(a) Lift curve

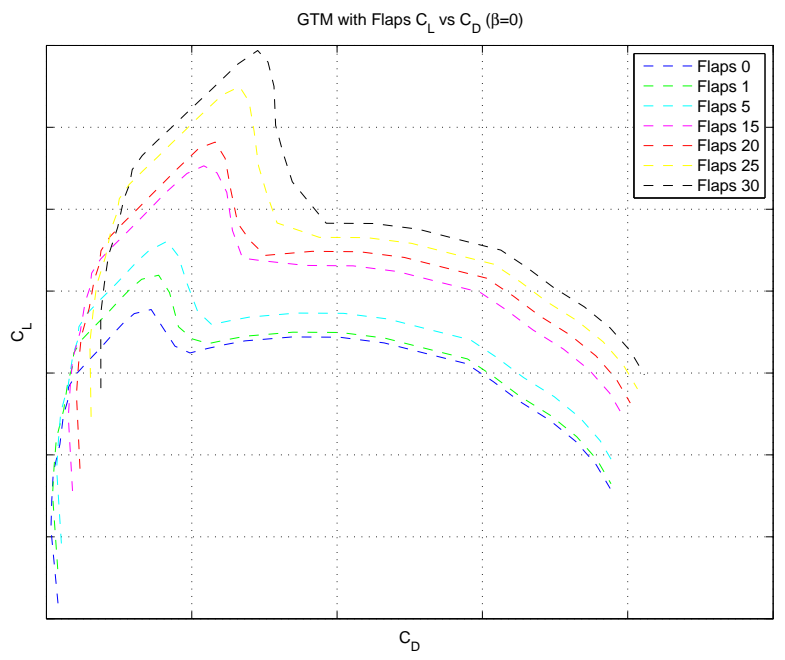

(b) Lift drag polar

Figure 4. Aerodynamic properties of the GTM model after Reynolds corrections for the full scale model. Note: most axes labels have been deleted because of the confidentiality of the model data. 


\section{Overview of Stall Recovery Guidance Algorithms}

This section describes the essentials of the two guidance algorithms that were evaluated in the Vertical Motion Simulator. More detailed descriptions are given in Ref. [22,23].

\section{A. Fast Model Predictive Control (FMPC)}

Model predictive control (MPC) is an advanced optimal control method that lends itself to the determination of scenario specific stall recovery guidance. The first step in the approach is to linearize and discretize the aircraft equations of motion around the current aircraft state of interest, defined as the airspeed, angle of attack, and pitch. This linear discrete model then approximately predicts the aircraft state trajectory from the current state, given a sequence of pitch control inputs. An optimal pitch recovery maneuver that returns the aircraft to the target state, is then found by solving a linearly constrained optimization problem with quadratic cost function (i.e., a quadratic program). In this formulation for stall recovery, the solution to the optimization problem determines the input pitch rate sequence required to recover the aircraft across a 30 second time horizon, discretized to 0.5 second increments a In the classical model predictive control approach, only the most immediate control input from the solution is used, and the entire computation is repeated in the next computational cycle (or simulator frame). To determine the guidance cue input, however, it was found that a blended combination of the pitch and pitch-rate error terms was easier to track, as judged by a test pilot subject matter expert.

The actual online computation of the maneuver ultimately requires solving a quadratic program, which is accomplished using the Fast Model Predictive Control (FMPC) interior point algorithm developed in [28. This algorithm has important features that help to ensure fast and reliable convergence of the optimal solutions at relatively low computational cost suitable for online implementations. The more advanced computational details covering the application of the FMPC algorithm to stall recovery guidance are documented in 23. There are two important features to the FMPC approach. The first is that the optimal recovery input is recomputed from the current aircraft state in each simulator frame (or computational cycle). This means that the pilot is always provided with optimal guidance from the current aircraft state (with respect to the quadratic cost function), independent of how well he or she follows that guidance. The second important feature is that constraints on the state variables (airspeed, angle of attack, pitch angle and rate) can be specified directly. In particular, constraints on the pitch rate were used to prevent exceeding load factor limits, while the angle of attack corresponding to stall warning was used as the maximum angle of attack constraint in the formulation. The optimal solution with the predictive model then intelligently includes consideration for protecting the load factors and angle of attack during the full pull-up phase of the recovery maneuver.

Figure 5 illustrates these important features, by showing the predictive guidance maneuvers, as well as the discretized aircraft trajectory flown by one of the pilot participants in the study. In this scenario, the autopilot flies the aircraft into a deep aerodynamic stall, at 40,000 feet. At $\mathrm{t}=0$ seconds, the manual piloted recovery begins with the model predictive guidance. The gray trajectories show the computed predictive recoveries at each time sample $\mathrm{b}$ Initially, while the angle of attack exceeds the stall warning angle of attack, the default $5 \mathrm{deg} / \mathrm{sec}$ pitch down guidance is provided to reduce the angle-of-attack — because the linearized model used for the FMPC computation is invalid in the stalled dynamics regime. In the plots, the pilot follows the default pitch guidance below the PLI (pitch limit indicator) position, and therefore reduces the angle of attack below the stall warning threshold. At this point, around 4 seconds into the recovery the model predictive guidance becomes valid and initially finds a pitch input plan that pulls the aircraft pitch to 30 degrees nose-down. The pilot lags the guidance, following it only to about 15 degrees nose-down, at which point the pilot follows the guidance to pull the nose of the aircraft back up. At each time instance, the predictive guidance intelligently re-schedules the pull-up maneuver to efficiently use the full angle of

\footnotetext{
aThe 30 second time horizon was about the shortest time required to cover the important duration of the push and pull-up phases of the longest stall recoveries at high altitude.

${ }^{\mathrm{b}}$ Actually, only a small sub-sample of the computed trajectories are shown. The actual predictive guidance computation completes with each simulator frame, at 50 frames per second.
} 

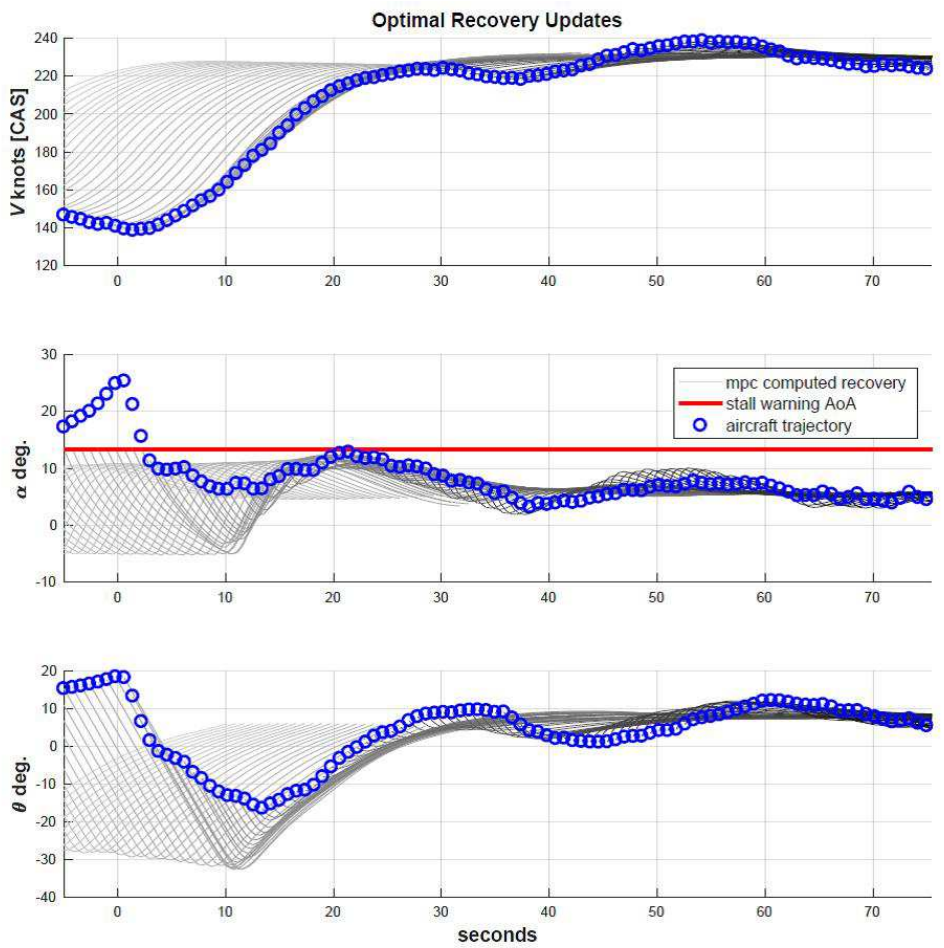

Figure 5. Model predictive control based guidance strategy for a stall recovery maneuver.

attack margin, just grazing the stall warning limit around 20 seconds into the recovery, and ending up at the target airspeed. The results of this study show, that the aggressive guidance computed by this algorithm significantly improves the safety of the piloted recoveries with respect to secondary aerodynamics stalls in the high altitude deep-stall scenario highlighted in this example. Essentially, the guidance uses the aircraft flight dynamics to compute how far to pitch nose-down in the initial recovery, at what airspeed to begin pitching up, and the pitch up rate that smoothly returns the aircraft to the target airspeed, all in one smooth maneuver. In this initial study the aim was to keep the FMPC problem formulation as simple as possible. This was done in an effort to achieve the rapid and reliable convergence needed to run the algorithm well within the $50 \mathrm{~Hz}$ simulator loop on the Vertical Motion Simulator (VMS) computer. One of the benefits of the overall approach, however, is that it readily supports additional constraints on pitch angle and airspeed, and more sophisticated equations-of-motion can also be incorporated into future implementations. To a large extent, the FMPC algorithm can also be configured to work within computational time limits. The algorithm can split the full convergence computational cost across multiple simulator frames if needed, and it can provide a sub-optimal, but feasible, guidance solution if it needs to be stopped before full convergence is achieved.

\section{B. Energy Based Algorithm (EBA)}

Another approach for computing stall recovery guidance uses a physically motivated energy tradeoff analysis to derive the control guidance, while maintaining load factor limits and protecting against secondary stall.22 Ref. 29 - 31 show previous research in flight control based on energy principles. The benefits of this approach are that the computational cost is significantly less than any optimal control method, it does not necessarily require any estimated model information, and it is, therefore, easily implemented for online use.

The main purpose of the stall recovery strategy advised by the FAA for flight crew training, as discussed 
in Section II is to reduce the angle of attack and increase the airspeed. This can be accomplished most rapidly by trading potential energy (altitude) for kinetic energy (speed), as efficiently as possible. The only complicating factors are that, during the trade-off process, energy is dissipated through drag $D$, which requires the application of thrust $T$ over time. The FAA recovery template implicitly uses these factors when it advises a pitch down to reduce the angle of attack (and the drag), beginning the trade-off between altitude and airspeed, and adding thrust carefully to avoid pitch trim related upsets.

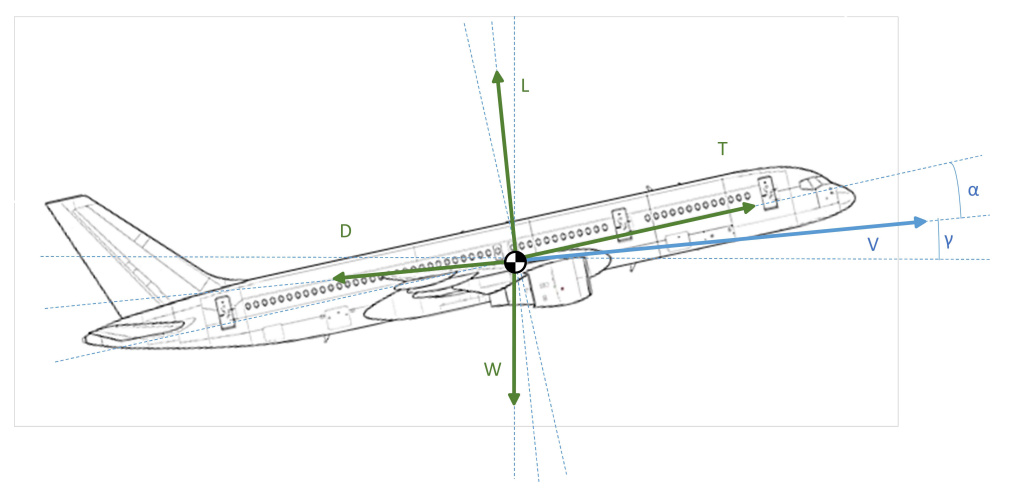

Figure 6. Acting forces on the aircraft model

From the balance of the forces lift $L, \operatorname{drag} D$, thrust $T$, and weight $W$ in Figure 6 , along with Newton's second law, applied to the direction of the velocity vector, one finds:

$$
\dot{V}+g \sin \gamma=\frac{T \cos \alpha-D}{m},
$$

where $\alpha$ is the angle of attack and $\gamma$ the flight path angle. The specific energy rate can then be defined as:

$$
\dot{E}_{\mathrm{s}}=\frac{\dot{E}_{\mathrm{tot}}}{m}=g \dot{h}+V \dot{V}=V(\dot{V}+g \sin \gamma)=V\left(\frac{T \cos \alpha-D}{m}\right) .
$$

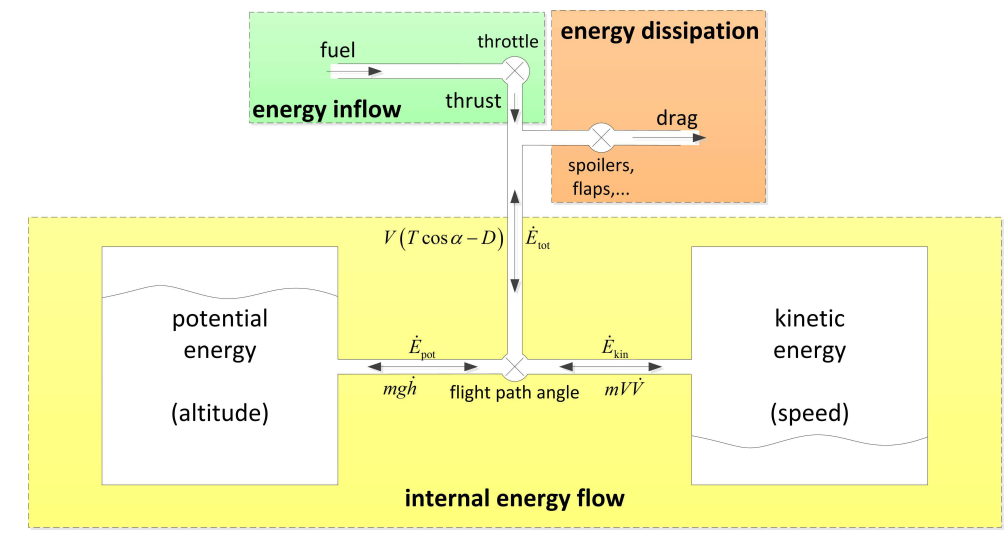

Figure 7. Energy reservoir analogy

The physical intuition behind Eq. (2) is illustrated by the reservoir analogy shown in Figure 7 Here, a direct exchange between potential (altitude) and kinetic energy (speed) is possible by controlling the flight path angle $\gamma$. Figure 7 shows that the throttles add to the total energy of the aircraft system, while drag causes some of the total aircraft energy to dissipate. The flight path angle (as controlled by the elevator) then regulates the net influx of total energy into the kinetic and potential energy reservoirs or vice versa. 
As a consequence, stall recovery is most efficient when one simultaneously dives, driving $\gamma<0$, and increases the thrust. As soon as enough speed has been gained, a pull up maneuver should be performed in order to establish level flight. During this pull up maneuver, secondary stall and load factor limits should be avoided. This drives the constraints for angle of attack: $\alpha<\alpha_{\text {stall }}$ and load factor: $n_{L F_{\min }}<n_{L F}<n_{L F_{\max }}$.

The EBA guidance law is derived essentially by solving Eq. (11) for the flight path angle required to achieve a desired acceleration

$$
\begin{aligned}
\gamma_{\text {guidance }} & =\arcsin \left(-\frac{1}{g}\left[\dot{V}_{\text {required }}-\frac{T \cos \alpha-D}{m}\right]\right) \\
& \stackrel{1}{=} \arcsin \left(-\frac{1}{g}\left[\dot{V}_{\text {required }}-(\dot{V}+g \sin \gamma)\right]\right)
\end{aligned}
$$

where the required speed rate $\dot{V}_{\text {required }}$ is defined as:

$$
\dot{V}_{\text {required }}=\frac{1}{\tau_{V}} \Delta V_{\text {required }}=\frac{1}{\tau_{V}}\left(V_{\text {target }}-V_{\text {current }}\right) .
$$

In this expression, $V_{\text {target }}$ is the airspeed target, and $\tau_{V}$ is an associated speed control time constant. The time constant $\tau_{V}$ determines the responsiveness of the speed guidance: the smaller the value for $\tau_{V}$, the more aggressive the dive will be in order to pick up speed.

Equations (3) and (4) provide the energy based guidance strategy calculation in two equivalent ways, depending on the prevailing conditions. Equation (44) does not require any aircraft model information, which makes the guidance independent of the aircraft model and can thus be applied on different aircraft types in a relative straightforward way. It does, however, require sensor values for the acceleration $\dot{V}$, which is especially sensitive to turbulence and needs additional filtering. As an alternative, Equation (3) can be used, which includes values for thrust $T$ and drag $D$. Thrust can be approximated based on the engine RPM $N 1$, but aircraft model information is needed for the drag $D$. For the study detailed in this report, the latter model based calculations were used.

Finally, constraints can be enforced directly on $\gamma_{\text {guidance }}$ as well as $\dot{\gamma}_{\text {guidance }}$ to protect against secondary stalls and exceeding load factor limits respectively:

$$
\begin{aligned}
\gamma_{\max } & =\gamma+\left(\alpha_{\max }-\alpha-\varepsilon_{\gamma}\right) \\
\dot{\gamma}_{\max } & =\frac{g}{V_{T}}\left(\Delta n_{z_{\max }} \cdot \cos \phi-\cos \gamma\right) \\
\dot{\gamma}_{\min } & =\frac{g}{V_{T}}\left(\Delta n_{z_{\min }} \cdot \cos \phi-\cos \gamma\right)
\end{aligned}
$$

where: $\Delta n_{z_{\max }}=2.5-\varepsilon_{n_{z}}$ and $\Delta n_{z_{\min }}=-1+\varepsilon_{n_{z}}$, where the load factor buffer $\varepsilon_{n_{z}}=0.2 \mathrm{~g}$ and another robustness buffer $\varepsilon_{\gamma}=2 \mathrm{deg}$ has been incorporated as well.

Furthermore, in order to achieve a smooth guidance signal in the presence of turbulence, additional first order filtering is needed for the calibrated airspeed (filtering frequency $\omega_{V}=2 \mathrm{rad} / \mathrm{s}$ ) and its time derivative (filtering frequency $\omega_{\dot{V}}=4 \mathrm{rad} / \mathrm{s}$ ). Additionally, a limit of $10 \mathrm{deg}$ for the maximum deviation of the guidance signal compared to the current state was included, since this deviation is the primary driver of how aggressive the pilots fly. More details about this algorithm can be found in Ref. 22 .

\section{Experiment Method}

The method for the piloted evaluation was based on standard procedures for human factor experiments. Usefulness evaluations were done after each run, combined with a short workload questionnaire. For the test pilots, Cooper Harper Handling Quality ratings $52 \mid 33$ were collected as well. A more extensive questionnaire

\footnotetext{
${ }^{\mathrm{c}}$ One possibility is using a blending of airspeed at lower frequencies and ground speed for higher frequencies.
} 
was given at the end of the study, which included a survey to determine a rating on the system usability scale. ${ }^{34}$ The number of pilots and repetitions were sufficient to observe certain trends in the data, but in most cases insufficient to warrant high statistical confidence in the results. Overall, the experiment objectives were to evaluate the usefulness of the guidance and the possible impact on pilot performance during the stall recovery. More concretely, the aim of the experiments was to verify if the following research hypotheses were satisfied:

Primary: Guidance improves pilot stall recovery performance, specifically, by promoting:

- appropriate application of pitch down to effectively eliminate aerodynamic stall, but not in excess of what is needed to recover safely

- gentle pitch up maneuvers to avoid secondary stalls during the pull up phase

- recovery at sufficient airspeed on the front side of the power curve

- reduced tendency to observe pilot induced oscillations in the recovery

- prevention of elevator saturation caused by excessive nose up stab trim

- gentle pitch rates to protect the load factor from exceeding safety limits

Secondary: Unguided stall recovery performance is improved after training with stall recovery guidance.

\section{A. Technologies}

The baseline condition for comparison was the conventional control setup, where the pilots had to fly the stall recovery maneuver without guidance, based on their individual airline training background and in accordance with the FAA template as discussed in Table 1. The control set up was such that the pilot could manually maneuver the aircraft by means of the control column and wheel, much like the conventional manual flying strategy for a civil airliner similar in size and behavior as a Boeing 757 class of aircraft. The aircraft was flown open loop without any stability and control augmentation system, but with a yaw damper. The pedals were used little during the experiments. During the evaluations, the aircraft was flown with and without guidance. In the latter configuration, the pilots could develop their own recovery strategy where they could choose their own recovery targets such as initial pitch down target angle, at which airspeed to begin pitching up and the pitch up rate without causing secondary stalls. The former configuration included guidance through the crosshair (also called split cue) flight director on the primary flight display, as well as a novel guidance feature for the throttle setting called 'throttle director'. In total, four scenarios were considered in the experiments: high altitude deep stall, low altitude with bank with and without excessive nose up trim, and descent for final approach. These scenarios were flown in a randomized order, with half of the pilots first flying without guidance and the other half first with the newly developed guidance algorithm under investigation. Each of the eight possible combinations of scenario and configurations (with and without guidance) was flown three times per pilot, resulting in a total of 24 runs per pilot. Since previous tests had shown that a significant learning curve develops throughout the runs, only one guidance algorithm was evaluated per pilot. At the start of the session, the pilot was given some time to familiarize himself with the simulator, experiment procedure, and performance rating metrics.

\section{B. Apparatus}

The evaluation was performed in the vertical motion simulator (VMS) at NASA Ames Research Center. The vertical motion simulator is the ideal facility to simulate stall scenarios and to test the recovery guidance technology's effectiveness because of the VMS's large motion envelope. The VMS motion system, shown in Fig. 8, is an uncoupled six-degree-of-freedom research motion simulator, with different interchangeable cockpit cabs and configurable flight deck instrumentation systems, wide-view outside visual display systems, electric control loaders and a high fidelity motion system. It is located in, and partially supported by, a specially constructed $120 \mathrm{ft}$ tower. The cab, shown in Fig. 9 (outside) and Fig. [10 (inside), serves as the aircraft cockpit for this research project. The evaluation pilot occupies the left seat, with a test engineer on the right. More information about the simulator can be found in Ref. $35+37$. 

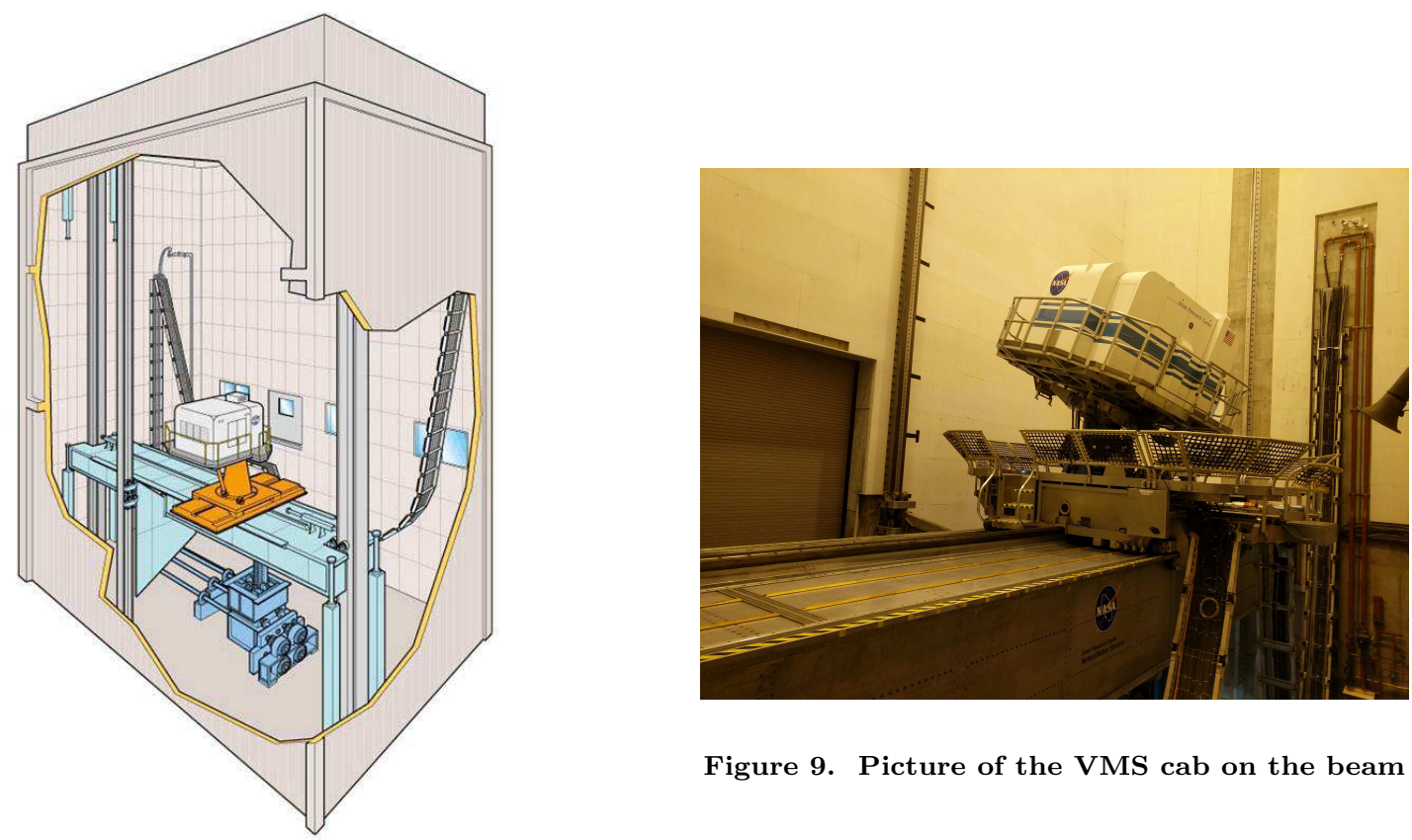

Figure 9. Picture of the VMS cab on the beam

Figure 8. Drawing with layout of the Vertical Motion Simulator

\section{Motion System}

The VMS motion system capabilities are provided in Table 2 . Included in the table are two sets of limits: system limits that represent the absolute maximum levels of attainable values under controlled conditions, and operational limits that represent attainable levels for normal piloted operations.

Table 2. Table of VMS motion system performance limits

\begin{tabular}{|c|c|c|c|c|c|c|}
\hline \multirow{3}{*}{$\begin{array}{l}\text { Degrees of } \\
\text { freedom }\end{array}$} & \multicolumn{2}{|c|}{ Displacement } & \multicolumn{2}{|c|}{ Velocity } & \multicolumn{2}{|c|}{ Acceleration } \\
\hline & System & Operational & System & Operational & System & Operational \\
\hline & limits & limits & limits & limits & limits & limits \\
\hline Longitudinal & $\pm 4 \mathrm{ft}$ & $\pm 4 \mathrm{ft}$ & $\pm 5 \mathrm{ft} / \mathrm{s}$ & $\pm 4 \mathrm{ft} / \mathrm{s}$ & $\pm 16 \mathrm{ft} / \mathrm{s}^{2}$ & $\pm 10 \mathrm{ft} / \mathrm{s}^{2}$ \\
\hline Lateral & $\pm 20 \mathrm{ft}$ & $\pm 15 \mathrm{ft}$ & $\pm 8 \mathrm{ft} / \mathrm{s}$ & $\pm 8 \mathrm{ft} / \mathrm{s}$ & $\pm 13 \mathrm{ft} / \mathrm{s}^{2}$ & $\pm 13 \mathrm{ft} / \mathrm{s}^{2}$ \\
\hline Vertical & $\pm 30 \mathrm{ft}$ & $\pm 22 \mathrm{ft}$ & $\pm 16 \mathrm{ft} / \mathrm{s}$ & $\pm 15 \mathrm{ft} / \mathrm{s}$ & $\pm 22 \mathrm{ft} / \mathrm{s}^{2}$ & $\pm 22 \mathrm{ft} / \mathrm{s}^{2}$ \\
\hline Roll & $\pm 18^{\circ}$ & $\pm 14^{\circ}$ & $\pm 52^{\circ} / \mathrm{s}$ & $\pm 40^{\circ} / \mathrm{s}$ & $\pm 229^{\circ} / \mathrm{s}^{2}$ & $\pm 115^{\circ} / \mathrm{s}^{2}$ \\
\hline Pitch & $\pm 18^{\circ}$ & $\pm 14^{\circ}$ & $\pm 52^{\circ} / \mathrm{s}$ & $\pm 40^{\circ} / \mathrm{s}$ & $\pm 229^{\circ} / \mathrm{s}^{2}$ & $\pm 115^{\circ} / \mathrm{s}^{2}$ \\
\hline Yaw & $\pm 24^{\circ}$ & $\pm 14^{\circ}$ & $\pm 52^{\circ} / \mathrm{s}$ & $\pm 46^{\circ} / \mathrm{s}$ & $\pm 229^{\circ} / \mathrm{s}^{2}$ & $\pm 115^{\circ} / \mathrm{s}^{2}$ \\
\hline
\end{tabular}

\section{Flight Deck Instrumentation}

The overall flight deck of the VMS was set up to resemble a simplified generic airliner cockpit as shown in Fig. 10. The installed hardware consisted of two aircraft seats, a wheel/column and rudder pedals, a control pedestal and seven liquid crystal display (LCD) screens to display the flight instrumentation $(60 \mathrm{~Hz}$ refresh rate). There was no mode control panel. The McFadden Force Loader system is used to deliver versatile, accurate, and realistic control cues to the pilot. With this system, control force feel characteristics 
- such as position trim, breakout, friction and non-linear gradient - can be easily tailored to meet the needs of the research program. The control pedestal is equipped with four backdriven throttle levers, as well as flaps and spoiler levers. Since the simulated aircraft is a twin engine plane, the two left and right throttle levers were pairwise bolted together for this experiment. The displays were based on the Boeing electronic flight instrumentation system and consisted of a primary flight display (PFD) including crosshair (split cue) flight director and the novel 'throttle director' as shown in Fig. 11] a conventional navigation display, and a simplified engine information display only showing N1 numbers. Pitch and roll commands were presented on the split-cue director. The 'white' throttles in Fig. 11 indicate an 'add throttle command' condition to move the white throttles to the magenta cutout in the right wing of the airplane symbol. At the end of each simulation run, a summary page of the performance metrics was shown to the pilots on the outermost displays where the electronic flight bag would be found in a regular state of the art airliner. A frequently heard comment throughout the simulation runs was that the view of the PFD was obstructed by the control wheel for large roll inputs.

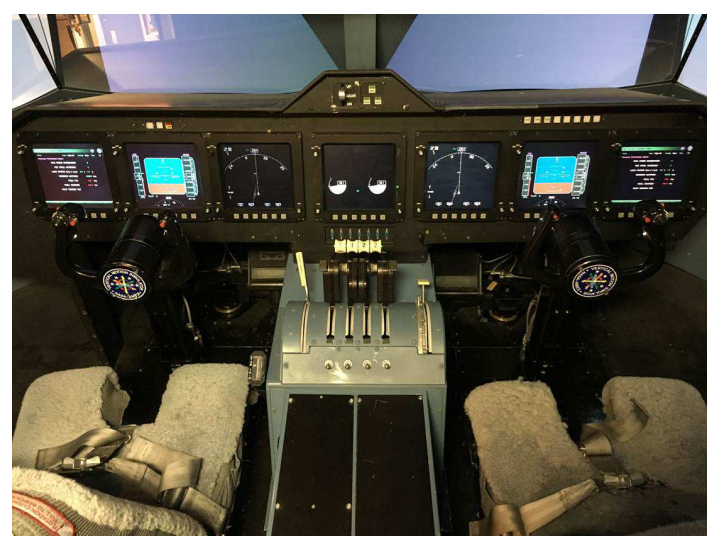

Figure 10. Cockpit setup for the SRG experiment in the VMS

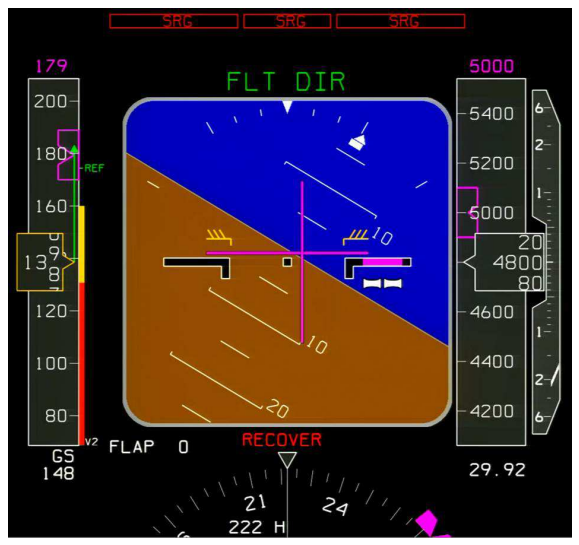

Figure 11. Primary Flight Display with Stall Recovery Guidance cues

\section{Outside Visual System}

The out-the-window visual system for the six-window collimated display is a Rockwell Collins EPX-5000. The outside visual scene gives the pilot attitude information and induces a sense of motion through the virtual world. Six vertically mounted cathode ray tubes produce computer generated images, which were viewed by the pilots through collimating mirrors. The resulting visual has a field of view of $110 \times 30 \mathrm{deg}$ with a resolution of $1400 \times 1050$ pixels per projector. The update rate of the visual system was $60 \mathrm{~Hz}$. For this evaluation, a reasonably accurate visual representation of San Francisco International Airport and the surrounding area was used. This is only relevant for the final approach scenario and the training runs, since the high as well as low altitude scenarios are in the clouds and lack any outside visual references. This makes the latter scenarios more challenging in the context of stall entrance and recovery.

\section{Participants}

Familiarity with the flown aircraft is one of the main requirements for the participants in a piloted evaluation. On the other hand, some flight test or evaluation experience is also beneficial, especially when using standard rating scales. Therefore, in this campaign, 30 experienced airline pilots from various major US airlines participated in the evaluation, which were between 36 and 68 years old, with an average age of 55 years. Also 10 test pilots from Armstrong Flight Research Center participated. The test pilots were specifically asked to evaluate handling qualities using the Cooper Harper rating scale. Over the entire group of participants, 
their flight hours were between 1,800 and 30,000 hrs, with an average of 13,900 hrs. All of the commercial pilots were current on a Boeing commercial aircraft with glass cockpit.

\section{Measures}

The guidance algorithms were assessed on two main types of dependent measures: implementation and operational.

\section{Implementation}

One measure of an algorithm's practical applicability is the computational load on the flight control computer. The amount of additional calculations necessary for stall recovery guidance must be sufficiently low to enable actual introduction within the near-term future. Especially possible implementation as a retrofit into the existing fleet poses additional challenges on the maximum allowable computational load. Table 3 shows some relevant values.

Table 3. On board processors for flight control computers in different airliner models

\begin{tabular}{lccc}
\hline \hline model & processor & speed & first flight \\
\hline Airbus A330/A340 & AMD486 & $32 \mathrm{MHz}$ & 1991 \\
Boeing 777 & HI-29KII & $50 \mathrm{MHz}$ & 1994 \\
Airbus A380 & PowerPC & $66-98 \mathrm{MHz}$ & 2005 \\
Boeing 787 & PowerPC 750FX & $166 \mathrm{MHz}$ & 2009 \\
\hline \hline
\end{tabular}

The Vertical Motion Simulator platform selected for the study used a DEC-Alpha computer running a Real-Time Operating System at $1.25 \mathrm{GHz}$. The frame rate of the simulation was $50 \mathrm{~Hz}$, i.e. $20 \mathrm{~ms}$ per frame. For this study, it was a requirement for both algorithms to complete the guidance computation along with the simulator model calculations within this $20 \mathrm{~ms}$ limit. The two guidance algorithms in this study fall on opposite ends of the computational cost spectrum. The Energy Based Algorithm uses an analytical calculation that only requires a few lines of code to evaluate (with no loops or potential convergence issues). As such, it is probably about the least computationally demanding guidance algorithm possible. The Fast Model Predictive Control algorithm, on the other hand, is closer to the most computationally expensive algorithm that can be evaluated on the target platform and frame time limit. Figure 12 shows the distribution of the longest model execution time for each simulation run in the experiment $\mathrm{d}$ The plot on the left shows that the EBA algorithm adds negligible cost to the no guidance case. The plot on the right shows the FMPC performance, which takes from 3 to 10 times as long as the baseline model evaluation with no guidance. The FMPC algorithm also has significantly increased variation in evaluation time. This is caused by the difference in computation time to solve the FMPC optimization problem, through an iterative solver, from varying initial aircraft states. Still, the FMPC algorithm ran reliably without producing any frame overruns for the entire simulation experiment. Overall, this is good performance for an optimal control algorithm, especially since such algorithms are often not fast or reliable enough to work under the operating conditions required for this experiment.

\section{Operational}

The impact of the new technology was assessed by means of some operational dependent measures. The operational variables were concerned with the interaction between the new technology and the pilot. Objective (for example, measured pilot control activity) as well as subjective (for example, workload assessment) variables were measured.

\footnotetext{
dThere are thousands of model evaluations per run (e.g., 3000 model evaluations in a 60 second run). Each of the time measurements used to create Figure 12 was the worst-case model evaluation time that occurred during a run.
} 


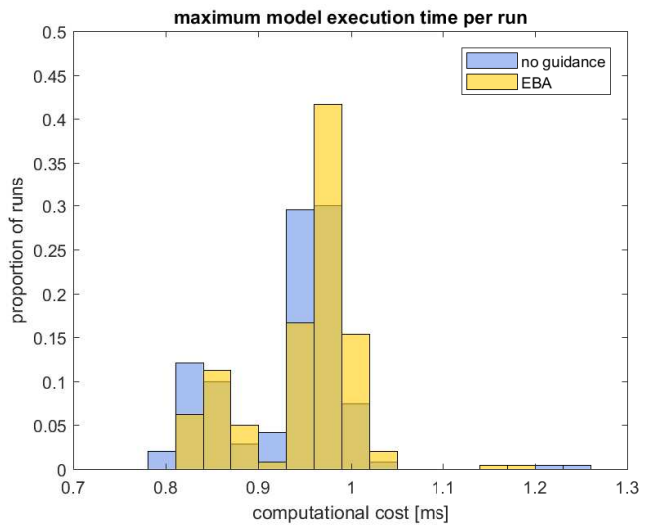

(a) Computational load without guidance and for EBA algorithm

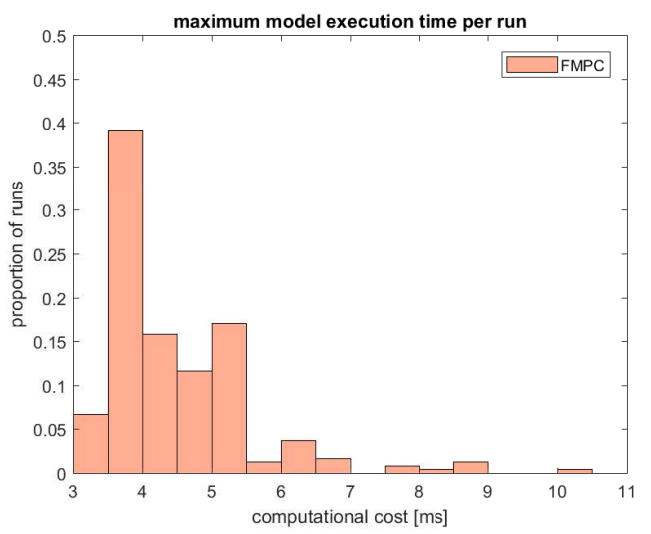

(b) Computational load for FMPC algorithm

Figure 12. Maximum computational load per run over all scenarios

\section{Objective Measures}

The objective measurements consisted of the pilot activity, measured by the root mean square of the control column and wheel deflection rate, and some selected metrics of the aircraft, which depended on the type of pilot. For commercial pilots emphasis was placed on metrics such as number of secondary stalls and maximum speed exceedances, and extreme load factor values. Altitude loss was considered as well, but only to the extent that it should not be excessive. Specifically for the test pilots, tracking of the guidance signal also became important for the Cooper Harper Handling Qualities ratings. Therefore the following guidance metrics were used: pitch flight director capture time, pitch track error, and accrued time that the throttle error (with respect to the guidance) exceeded 25\%. As a form of instant feedback to the pilot about their performance, the numbers for these metrics were shown on the outer cockpit displays and appropriately color coded to distinguish between desirable (green), adequate (yellow) and inadequate (red performance) after each run. Screenshots of how these metrics were presented can be found in Fig. 13. The numbers for these metrics can be found in Tables 4 to 6

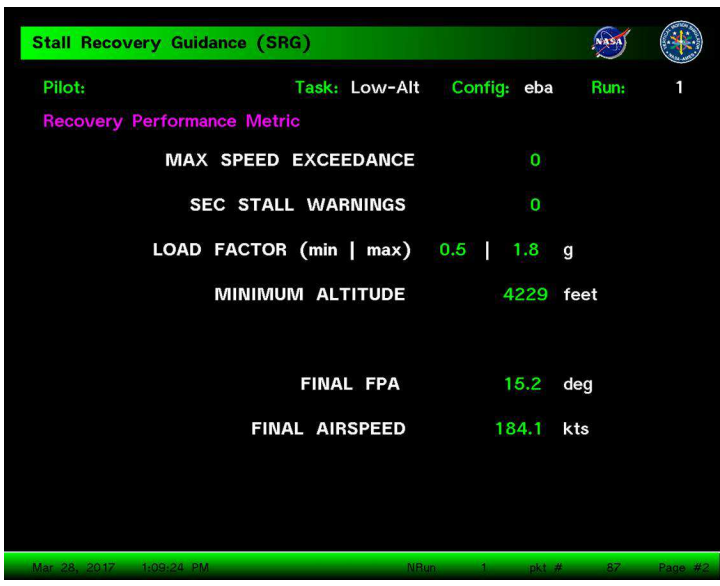

(a) Metrics for commercial pilots, all desirable

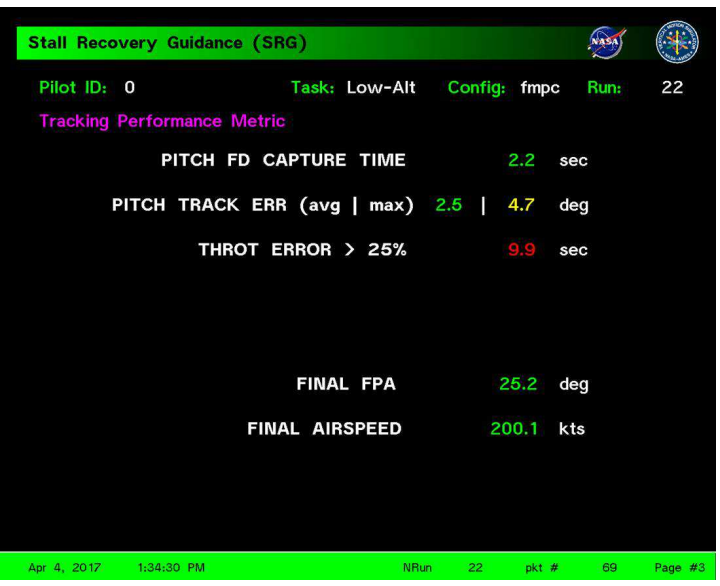

(b) Metrics for test pilots, desirable (green), adequate (yellow) as well as inadequate (red)

Figure 13. Performance metrics for commercial and test pilots with appropriate color coding 
Table 4. Performance Criteria for high altitude (HAS), low altitude (with or without excessive trim) (LAS) and approach (APS) scenarios, for the commercial pilots

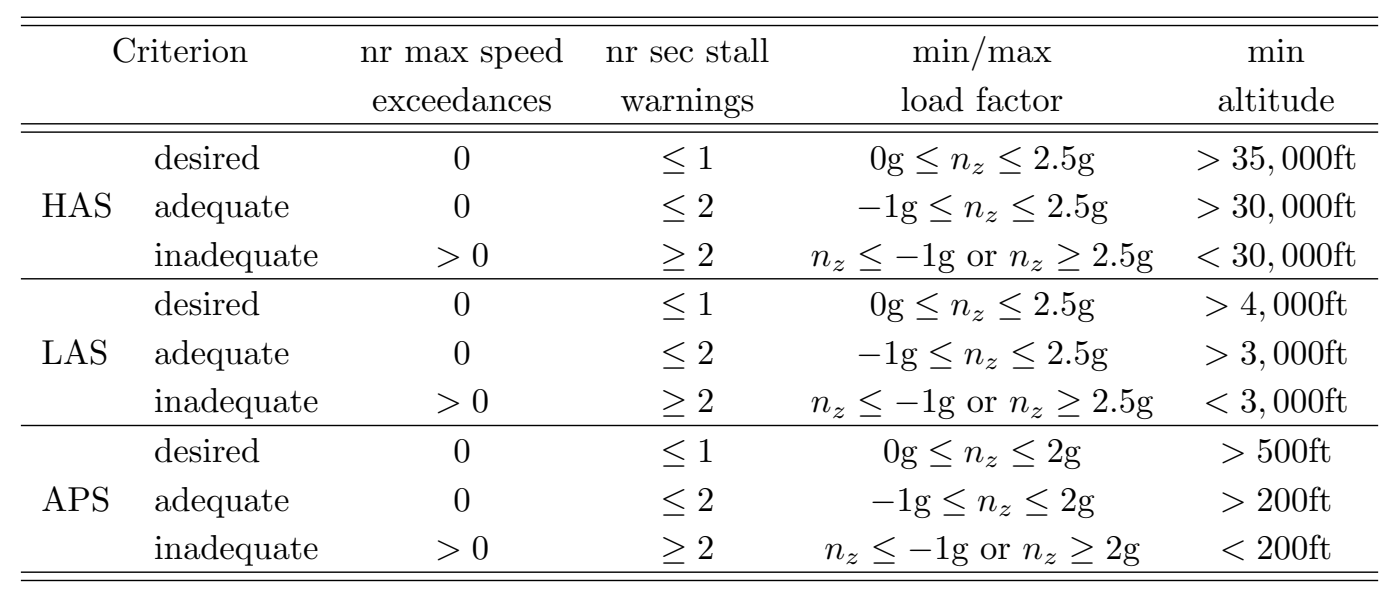

Table 5. Performance Criteria for high altitude (HAS), low altitude (LAS), low altitude with excessive trim (LANUS) and approach (APS) scenarios, for the test pilots

\begin{tabular}{llccc}
\hline \hline \multicolumn{2}{c}{ Criterion } & $\begin{array}{c}\text { Pitch FD } \\
\text { capture time }\end{array}$ & $\begin{array}{c}\text { Pitch tracking } \\
\text { error }\end{array}$ & $\begin{array}{c}\text { total time } \\
\text { throttle setting }>25 \%\end{array}$ \\
\hline \hline \multirow{3}{*}{ HAS/LAS/APS } & desired & $<3 \mathrm{~s}$ & $\leq 2.5 \mathrm{deg}$ & $<3 \mathrm{~s}$ \\
& adequate & $<6 \mathrm{~s}$ & $\leq 5 \mathrm{deg}$ & $<6 \mathrm{~s}$ \\
& inadequate & $>6 \mathrm{~s}$ & $\geq 5 \mathrm{deg}$ & $>6 \mathrm{~s}$ \\
\hline \multirow{2}{*}{ LANUS } & desired & $<3 \mathrm{~s}$ & $\leq 2.5 \mathrm{deg}$ & $<5 \mathrm{~s}$ \\
& adequate & $<6 \mathrm{~s}$ & $\leq 5 \mathrm{deg}$ & $<10 \mathrm{~s}$ \\
& inadequate & $>6 \mathrm{~s}$ & $\geq 5 \mathrm{deg}$ & $>10 \mathrm{~s}$ \\
\hline \hline
\end{tabular}

Table 6. Termination Criteria for Stall Recovery Maneuver

\begin{tabular}{lccc}
\hline \hline Criterion & final flight path angle & & final airspeed \\
\hline desired & $>-1 \mathrm{deg}$ & and & $>V_{\text {ref }}-5 \mathrm{kts}$ \\
adequate & $>-1 \mathrm{deg}$ & and & $>V_{\text {ref }}-10 \mathrm{kts}$ \\
inadequate & $<-1 \mathrm{deg}$ & or & $<V_{\text {ref }}-10 \mathrm{kts}$ \\
\hline \hline
\end{tabular}

\section{Subjective Measures}

Subjective measures consisted of workload, usefulness, handling qualities (test pilots only), and the systems usability scale (SUS) ${ }^{34}$ Assessment of the handling qualities was done by means of the Cooper Harper Rating Scale. 32,33

\section{E. Procedure}

Four scenarios have been defined with the purpose of bringing the experiment pilots to a stall condition. In all scenarios, the autopilot flew the aircraft into a stall for the purpose of consistency across all runs and pilots. The speeds were initialized slow so that the stall could be reached within half a minute. For all scenarios, the recovery objective is to recover to a stable unaccelerated flight from a fully stalled flight 
condition, with a flight path angle which should be non-negative, without excessive altitude loss, avoiding secondary stalls, and with no tendency to pilot induced oscillations (PIO) in pitch, roll or throttle control.

\section{High Altitude Scenario (HAS)}

This scenario was partly inspired by a recent series of high altitude stalls, such as Air France Flight AF447 and Air Algerie Flight 5017! ${ }^{7}$ The aircraft was initialized and trimmed at 170 KIAS and at a flight path of $-2.5 \mathrm{deg}$, cruise thrust setting for a clean aircraft near the altitude ceiling $(40,000 \mathrm{ft})$ in light turbulence. When the run starts, the autopilot attempts to hold altitude. Since the initial trimmed thrust setting is not changed, the aircraft starts bleeding speed. As a consequence, the autopilot flies the aircraft beyond the stick shaker into a full stall. For this scenario, the stall recovery triggers at an angle of attack of 25 degrees, well beyond the onset of aerodynamic stall. At this point, the autopilot and autothrottle disconnect and the pilot has to recover to a stable flight condition without excessive altitude loss using the pitch, roll and throttle flight directors when they are available, while trying to avoid secondary stalls and excessive load factors or maximum speed exceedances. Fig. 14 shows the trajectory for this scenario. One of the main challenges in this scenario is recovering to a final airspeed that is not on the back side of the power curve.

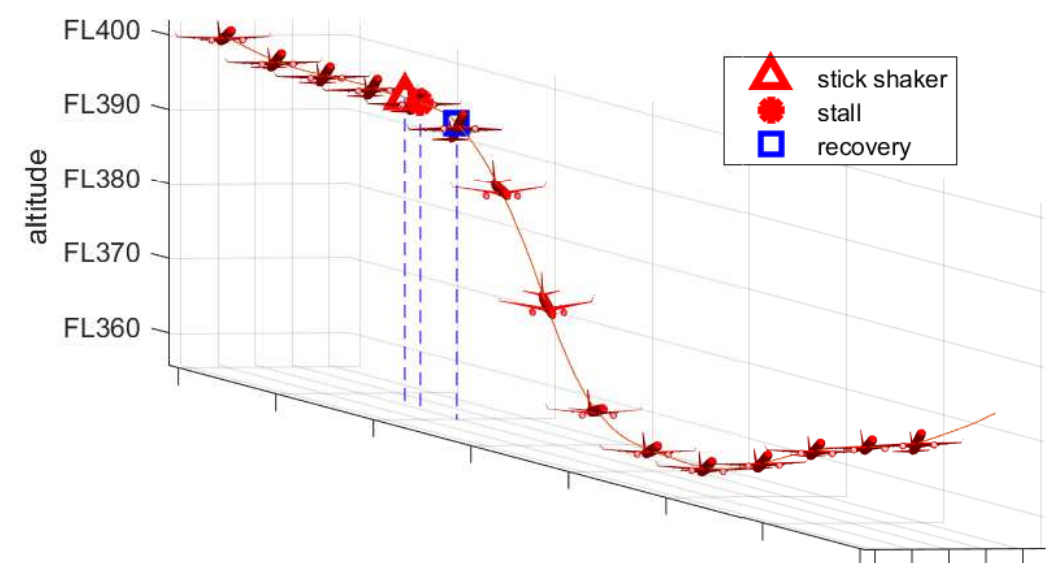

Figure 14. Typical trajectory of the high altitude scenario

\section{Low Altitude Scenario (LAS)}

In this scenario, the clean aircraft is initialized at $180 \mathrm{KIAS}$ in level flight at 5,000ft with light turbulence. The autopilot is in altitude hold mode and commands a $25 \mathrm{deg}$ bank to the left. Subsequently, the autothrottle commands the throttle to idle, causing the aircraft to bleed airspeed. Consequently, the autopilot flies the aircraft beyond the stick shaker into a full stall. In this scenario, the stall recovery is triggered right at the onset of aerodynamics stall, when the angle of attack exceeds its value corresponding with the maximum lift coefficient: $\alpha>\alpha_{L_{L_{\max }}}$, at this point the autopilot and autothrottle are disconnected. Also here, the pilot is required to recover to a stable flight condition without excessive altitude loss using the pitch, roll and throttle flight directors when they are available, while trying to avoid secondary stalls and excessive load factors or maximum speed exceedances. A typical trajectory for the low altitude scenario is shown in Fig. 15. The main challenge in this scenario is to follow the three guidance signals for pitch, roll and thrust simultaneously.

\section{Low Altitude with excessive Nose Up trim Scenario (LANUS)}

This scenario is similar to the previous one, but here the automation causes the stabilizer to trim for an excessive nose-up pitch. This results in a reduction of control authority in pitch. Especially combined with 


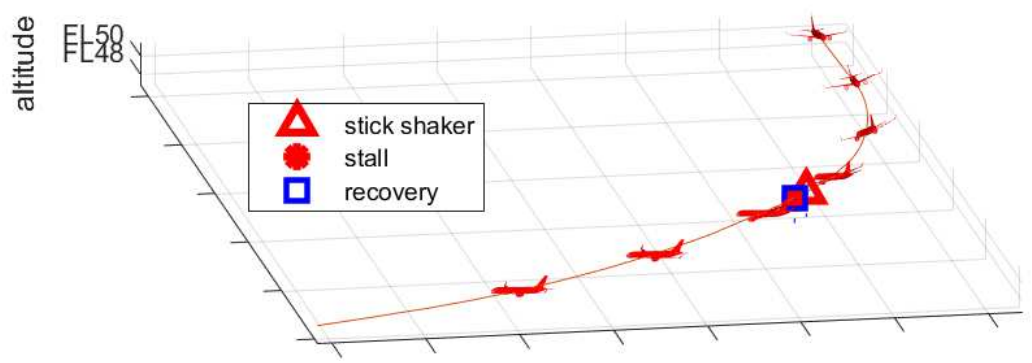

Figure 15. Typical trajectory for the low altitude scenario

full thrust (provided by engines mounted under the wings) during the stall recovery, the elevator is at risk of saturating. Manual adjustment of the trim stabilizer during the recovery will be needed to allow for sufficient control authority of the elevator. The main challenge in this scenario, in addition to the previous one, is avoiding loss of control authority in pitch by correctly modulating throttles and trim stabilizer, such that the elevator does not saturate.

\section{Approach Scenario (APS)}

In this scenario, the aircraft with full flaps and gear down is initialized at 140 knots airspeed, wings level at $1,100 \mathrm{ft}$ with a $700 \mathrm{ft} / \mathrm{min}$ descent rate in light turbulence. The autothrottle commands idle thrust while the autopilot attempts to hold the same flight path angle, causing the aircraft to bleed airspeed and bringing it into a full aerodynamic stall. Stall recovery triggers again at the onset of the aerodynamic stall, after the autopilot and autothrottle are disconnected. The pilot has to recover to a stable flight condition with minimum altitude loss (given its current altitude), while avoiding secondary stalls, excessive load factors or maximum speed exceedances (maximum flap speed for full flaps), using the pitch, roll and throttle flight directors when they are available. This accident was (partly) inspired by the Asiana Flight 214 accident at San Francisco. $\frac{38}{18}$ A typical trajectory is shown in Fig. 16] The challenges in this scenario are mainly the small altitude margin to speed up, combined with the narrow speed margin between stall speed and overspeed (for full flaps down configuration) which results in an increased risk for overspeed during the recovery maneuver.

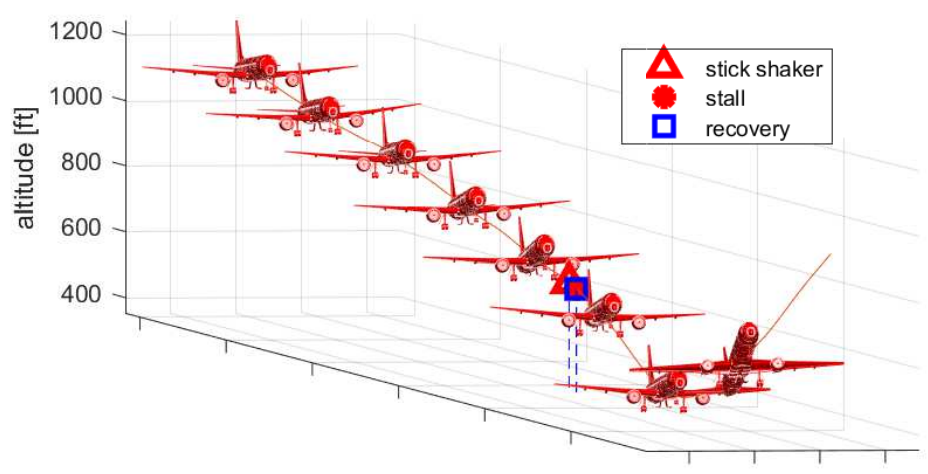

Figure 16. Typical trajectory of the approach scenario 


\section{Results}

This section discusses the analysis of the flight recovery performance data with respect to each of the scenarios. This paper focuses on the highlights of the data analysis. A more extensive and detailed discussion of the experiment results and pilot feedback can be found in Ref. 24. A convention for all the time histories in the results section is that the stall recovery maneuver starts at zero seconds in the plots. All the run data shown in this section focus on the first runs (out of three) of each pilot per scenario and per configuration, such that learning effects are eliminated as much as possible.

\section{A. High Altitude Scenario}

Generally, high altitude with significant post-stall behavior was the scenario where the performance difference with and without guidance became most apparent. Without guidance, pilots were usually more cautious during their recovery maneuver, resulting in shallower dives and less altitude loss, but also a higher risk that they would not successfully recover towards the front side of the power curve. Fig. 17] shows an illustrative example of one of these recovery trajectories with and without FMPC guidance. This scenario also showed the most significant difference in performance between both recovery algorithms. The FMPC algorithm commanded the aircraft to roughly -20 degrees pitch, before beginning the pull-up maneuver at greater airspeed. Being somewhat unexpected, not all pilots were as successful initially tracking this more aggressive maneuver. The EBA algorithm pushed the aircraft down to about -7 degrees pitch, before beginning a more gentle pull-up maneuver. Figure 18] shows a comparison of time histories between some important variables for all high altitude scenario runs with no guidance, FMPC and EBA guidance. Fig. 18(a) shows that both guidance strategies generally bring the pilots back to the front side of the power curve. EBA guidance, however, has more tendency towards secondary stalls than FMPC. Fig. 18(b), on the other hand, shows the more benign nature of the EBA guidance, resulting in a somewhat smaller altitude loss and significantly smaller load factors, well within the comfort levels. However, no structural load limits were violated during any of the runs.

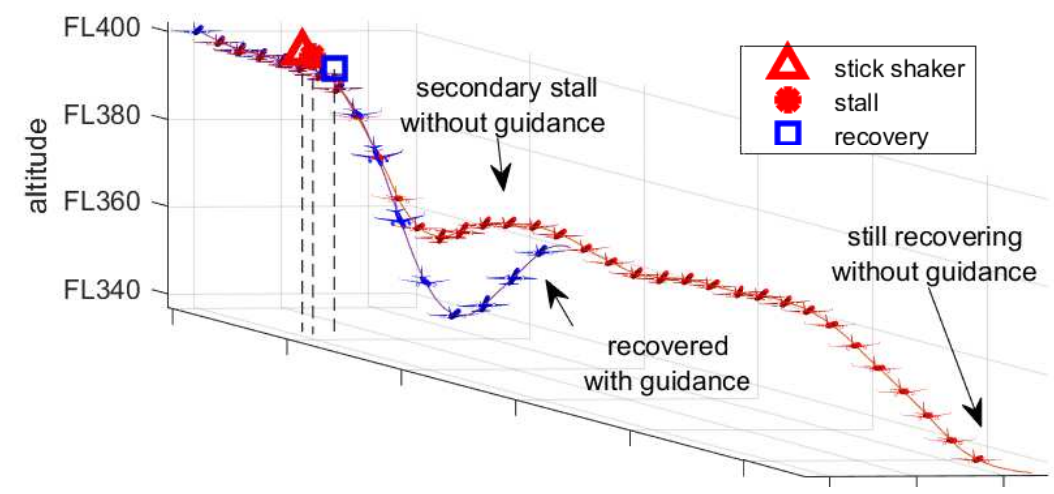

Figure 17. Example of recovery trajectory with and without FMPC guidance in high altitude scenario

Fig. 19(a) confirms the trends with respect to secondary stalls that could be observed in Fig. 18(a) FMPC is very effective in preventing secondary stalls as well as stick shakers. EBA has the tendency to lead the pilots in one secondary stick shaker and stall. However, it can be seen in Fig. 19(a) that both guidance algorithms are effective in preventing more than one secondary stick shakers and aerodynamic stalls, compared to the configuration with no guidance. Another important trend can be seen in Fig. 19(b) where it is shown that flying with guidance first (FMPC - 1) introduces a significant learning effect when one flies without guidance afterwards (no guidance - 2), as compared to flying with no guidance initially (no guidance - 1 and FMPC - 2). The reason for the improvement with FMPC is because the pitch down is significantly more aggressive. This helped the pilots to overcome their instinct to pitch up too early and risk secondary stall (due to decreased aerodynamic damping at high altitude). 

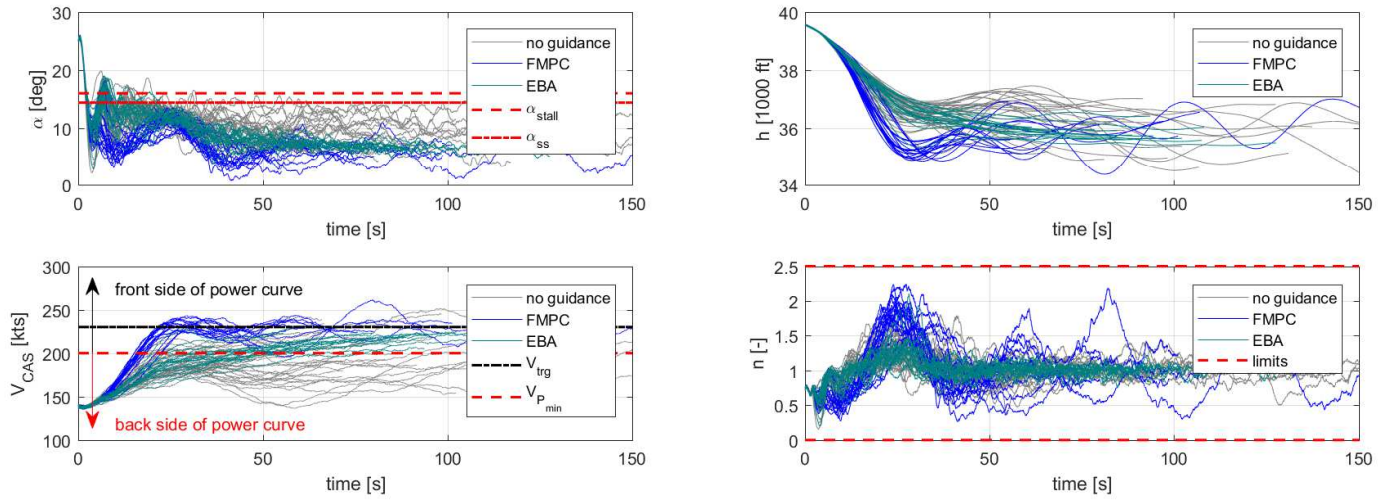

(a) Time histories of angle of attack $\alpha$ and airspeed (b) Time histories of altitude $h$ and load factor $n$ for all $V_{C A S}$ for all runs runs

Figure 18. Time histories of relevant quantities in the high altitude scenario
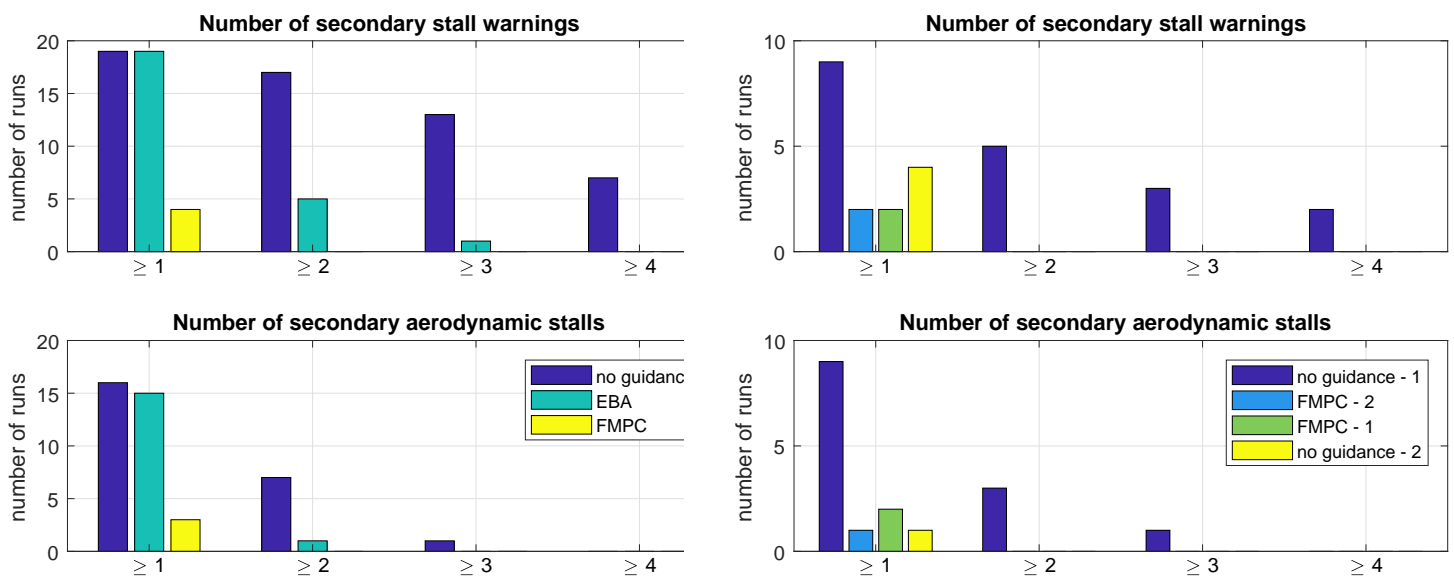

(a) Secondary stick shakers and secondary stalls w and without guidance

(b) Impact of learning effect on secondary stalls and secondary stick shakers

Figure 19. Impact of guidance and learning effect on secondary stick shakers and secondary stalls in the high altitude scenario 
Finally, a closer look at the back side of the power curve, of which the importance was already introduced before, can be found in the histograms shown in Fig. 20(a). Without guidance, more than half of the experiment pilots ended up on the back side of the power curve. With guidance, all runs ended on the front side of the power curve, except for one EBA run. Towards the end of this run, the pilot did not follow the guidance signal as accurately as the others. His motivation was that he 'did not need to in order to execute the recovery'. Fig. 20(b) shows the histograms for the total altitude loss over the complete recovery maneuver. Here it can be seen that both guidance strategies lead the pilots to somewhat more altitude loss compared to runs without guidance. This is exactly the energy trade off that needs to be made in order to get away from the back side of the power curve, which has a high priority in a high altitude scenario.
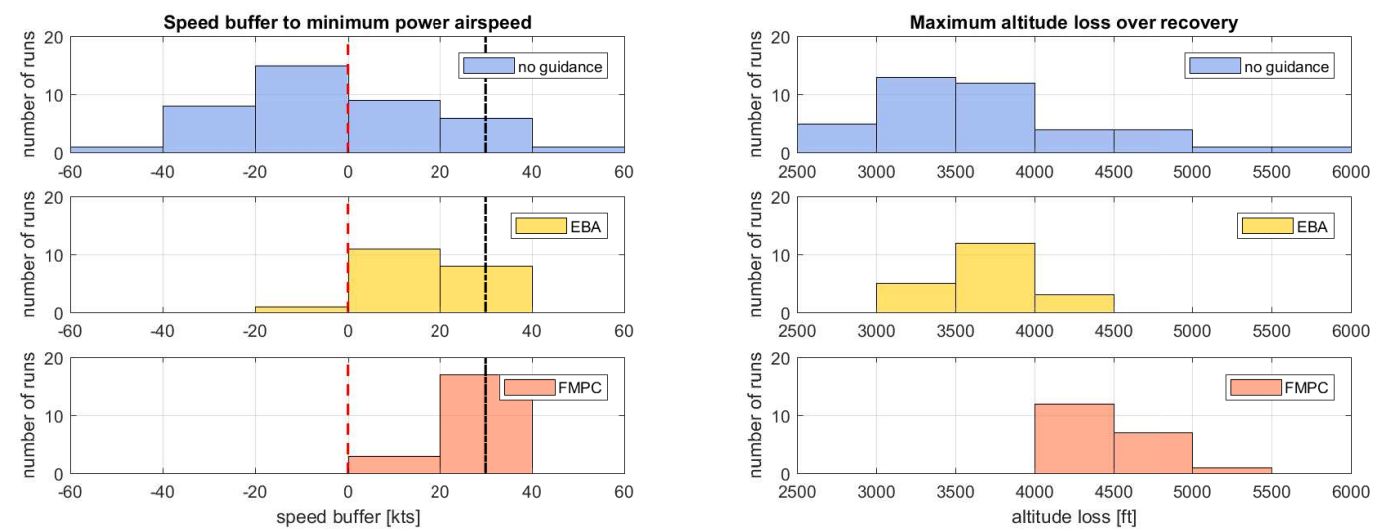

(a) Speed buffer to back side of power curve with and without guidance

(b) Total altitude loss with and without guidance

Figure 20. Impact of guidance on speed buffer and total altitude loss in the high altitude scenario

\section{B. Low Altitude Scenario}

The primary purpose of the low altitude stall recovery was to examine the effect of the guidance on a normal full stall recovery scenario, without proceeding to a significant post-stall behavior. Currently, pilots are trained to use the PLI (pitch limit indicator) to minimize their altitude loss during the recovery, sometimes at the expense of hitting multiple stick shaker warnings. Stall warnings may be acceptable as long as secondary stalls are avoided.

Figure 21 shows again a comparison of time histories between some important variables for all low altitude scenario runs with no guidance, FMPC and EBA guidance. Fig. 21(a) shows that both guidance strategies generally bring the pilots back to lower angles of attack and higher airspeeds. One can also see that some pilots needed more time to 'break the stall' (i.e. bring the angle of attack back to values below stall and stick shaker) without guidance. There is one significant outlier in the angle of attack time histories for FMPC towards the end of the recovery, but in this case the pilot stopped following the guidance at the end of the run. In the time responses for airspeed, it is not surprising to see that recoveries with guidance usually end up closer to the target speed. Fig. 21(b), on the other hand, shows the steep climb out for both guidance algorithms at the end of the recovery. This is needed to keep the aircraft with full thrust from accelerating. The load factors are very similar for both algorithms and well within structural as well as comfort load limits during all runs. Analyzing all the aforementioned time histories shows that EBA is marginally more aggressive in providing pitch down guidance than FMPC in this scenario.

Fig. 22(a) shows that EBA is effective in preventing secondary stalls as well as stick shakers, more than FMPC for the stick shakers, but to a lesser extent for the secondary stalls. Most probably, the explanation for this is the explicit safety buffer in EBA that keeps the guidance signal always three degrees below the PLI. Fig. 22(b) also shows that there is a much smaller spread in the maximum total altitude loss with 

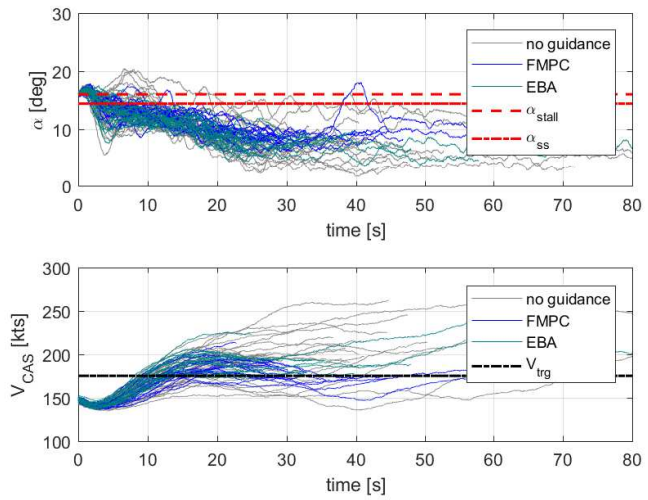

(a) Time histories of angle of attack $\alpha$ and airspeed $V_{C A S}$ for all runs
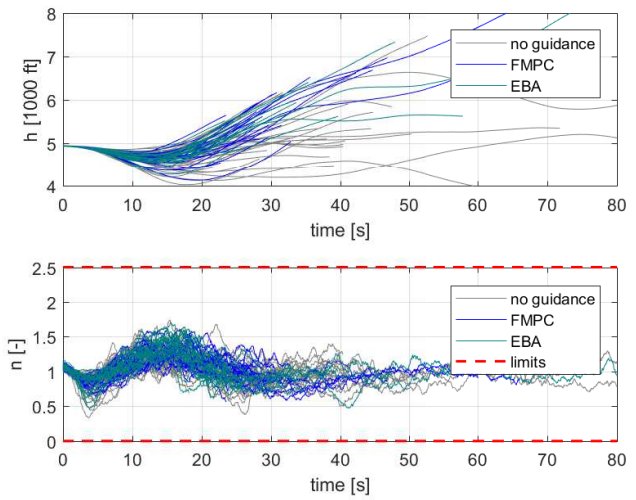

(b) Time histories of altitude $h$ and load factor $n$ for all runs

Figure 21. Time histories of relevant quantities in the low altitude scenario

guidance, which is consistent with the observation that the final airspeed with guidance usually converges closer to the target speed as observed in Fig. 21(a).
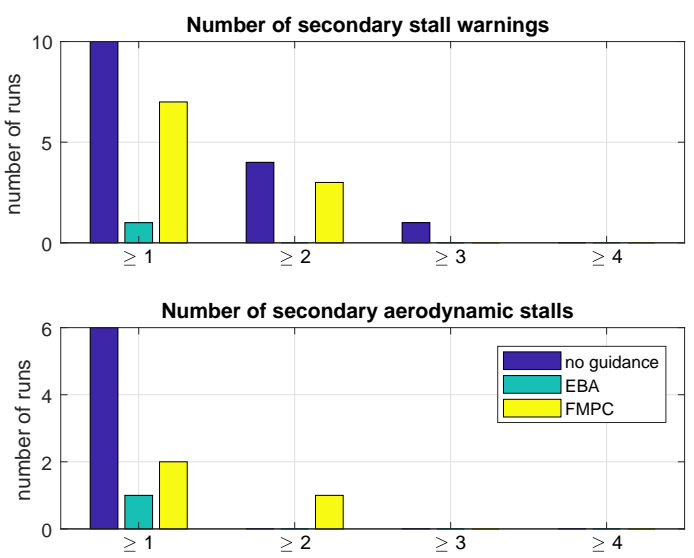

(a) Secondary stick shakers and secondary stalls with and without guidance
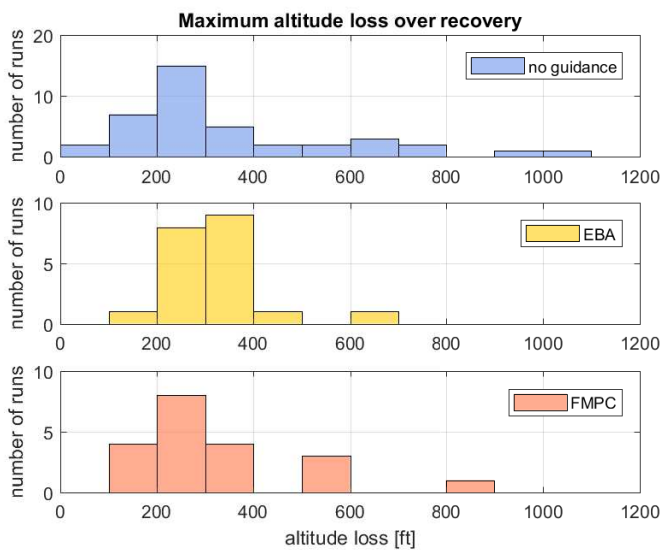

(b) Total altitude loss with and without guidance

Figure 22. Impact of guidance on secondary stick shakers, secondary stalls and total altitude loss in the low altitude scenario

\section{Low Altitude Scenario with Excessive Trim}

The low altitude stall with excessive nose-up trim scenario had the highest workload of the four scenarios. The setup for this scenario was exactly the same as for the low altitude scenario, except that excessive stabilizer trim to -9 degrees (instead of the usual -2 degrees for the regular low altitude scenario) was commanded while the aircraft decelerated towards stall. As a consequence, the autopilot provided increasing pitch down elevator deflection to compensate for this disturbance. This nose down command by the autopilot was removed, however, as soon as the system switched into manual recovery mode, which resulted in an initial pitch up tendency that increased the angle of attack significantly (usually exceeding 20 degrees). It typically took pilots around 1 to 3 seconds to arrest this tendency. This initial dynamical behavior is shown in the first 
few seconds of Fig. 23(a) Comparing the time histories in Fig. 23 for this scenario with the corresponding figure for the regular low altitude scenario (Figure 21), reveals overall that stall value exceedances for angle of attack are significantly larger and it also takes significantly longer to 'break the stall'. Figure 23(b) shows more loss of altitude but the load factors for this scenario were very similar and well within all limits. Further analysis of the results also showed that there was a direct connection between how fast the pilots adjusted the stabilizer to a less excessive setting and the total duration of the recovery maneuver.
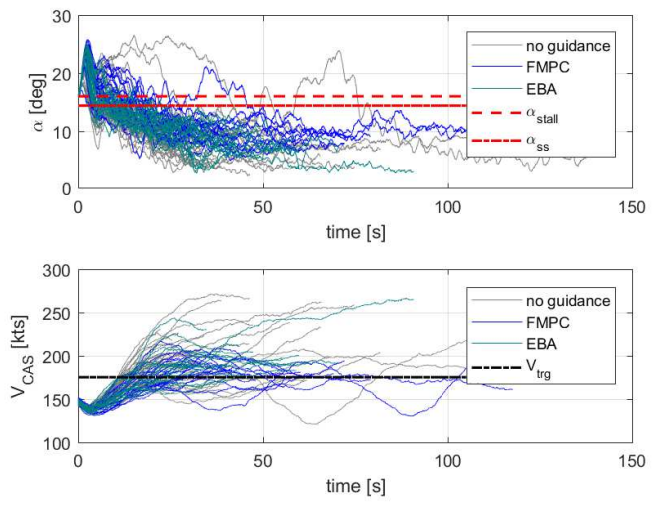

(a) Time histories of angle of attack $\alpha$ and airspeed $V_{C A S}$ for all runs
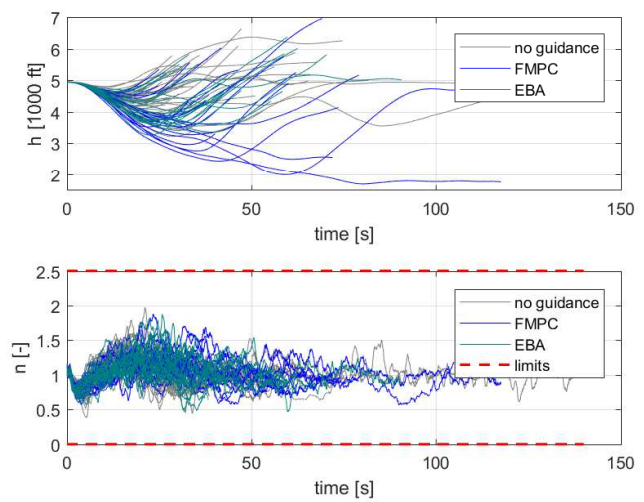

(b) Time histories of altitude $h$ and load factor $n$ for all runs

Figure 23. Time histories of relevant quantities in the low altitude excessive trim scenario

Figure 24(a) shows that guidance was not as successful in preventing secondary stall warnings and aerodynamic stalls as compared to the regular low altitude scenario (Fig. 22(a)). As a matter of fact, guidance seemed to exacerbate the number of stall warnings during the recovery, although EBA guidance showed a better trend for preventing more than one secondary aerodynamic stall. It is important to know that this is the only scenario where the thrust guidance deviated from the straightforward 'full throttle' guidance. For excessive trim, there is an upper limit value for thrust, above which the elevator cannot compensate the pitch up moment which is produced by the excessive stabilizer deflection and engines mounted under the wing. More information about how this upper limit is calculated can be found in Ref. 22 24. This meant that in this scenario, more than in any other one, pilots had to divide their attention between the pitch and roll flight directors on one hand and the throttle director on the other. From the pilot feedback it was clear that this was definitely a contributing factor to the number of secondary stalls. This same cause also had a significant impact on the total altitude loss during the maneuver. It can be seen in the histograms in Fig. 24(b) that, on average, guidance resulted in more altitude loss than no guidance, and the reason for this was because the guidance limited the use of the throttle.

The primary importance of the excessive nose up trim component of this scenario, however, was to see if the addition of throttle guidance could reduce the risk of saturating the elevator deflection margins, and therefore, the risk of an insurmountable pitch up tendency. The throttle guidance system was designed to compute the maximum permissible thrust, at the current stab position, that would not saturate the elevator deflections. This guidance was sometimes against the instinct of the pilot flying. Fig. 25(a) shows that the throttle guidance was indeed effective at getting the pilots to pull back the throttles while the aircraft was in an excessive nose up trim condition. The histogram was compiled from the throttle position traces for all the runs in this scenario, and the height of the bars represents the amount of time spent in that particular throttle bracket (out of a maximum of the first 10 seconds of each recovery). Overall, the figure shows that the participants flying with throttle guidance were more likely to keep their throttles at a safer level (below $50 \%$ ) while their trim stabilizer setting was exceeding -8 degrees. Fig. 25(b) compares the corresponding histograms for the elevator deflections obtained across all runs, in a similar way as for the throttle settings before. Without guidance, the participants spent significantly more time with an elevator deflection greater 

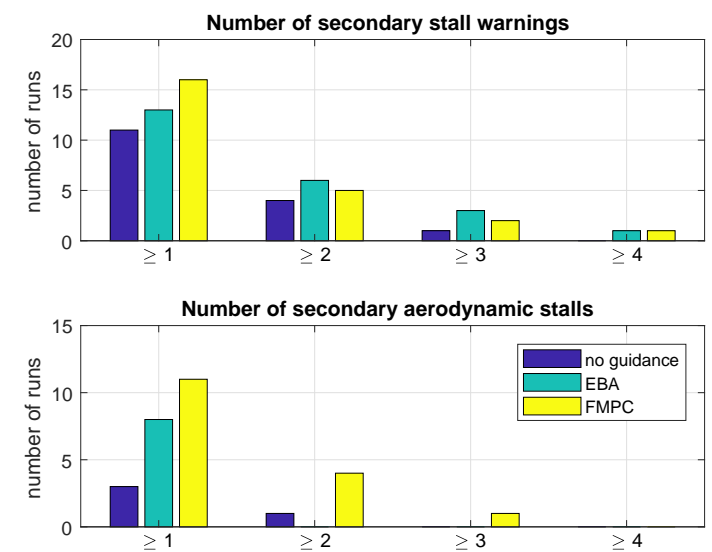

(a) Secondary stick shakers and secondary stalls with and without guidance
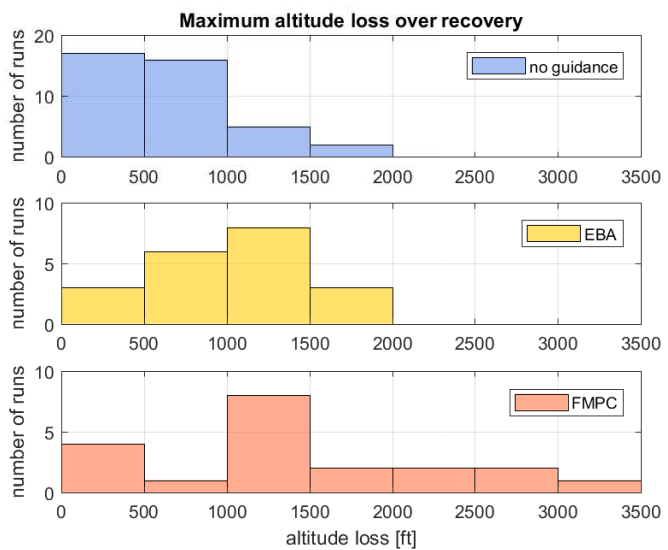

(b) Total altitude loss with and without guidance

Figure 24. Impact of guidance on secondary stick shakers, secondary stalls and total altitude loss in the low altitude excessive trim scenario

than 16 degrees (within 4 degrees of the upper saturation limit), than they did with guidance.

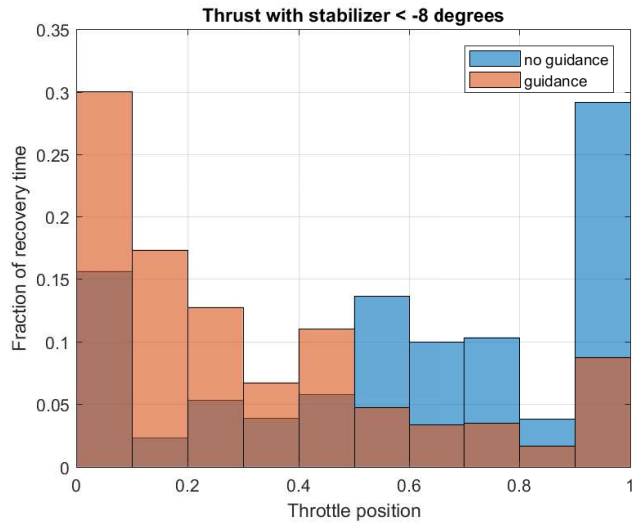

(a) Comparison of throttle deflection range with and without guidance

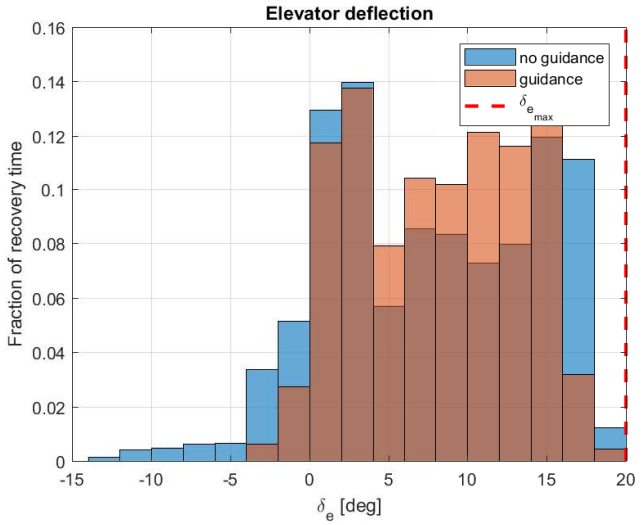

(b) Comparison of elevator deflection range with and without guidance

Figure 25. Impact of guidance on elevator and throttle deflection in the approach scenario

\section{Approach Scenario}

For the approach scenarios, there were no discernible differences in the piloted recoveries flown with the FMPC and EBA guidance algorithms. Both algorithms essentially matched most of the recovery maneuver that the pilots flew before seeing any guidance. This result can be observed in the time histories as shown in Figure 26. Fig. 26(b) shows that in all three categories the group of pilots achieved about the same mimimum altitude. Two outliers can be seen, where pilots were not capable to meet the minimum altitude constraint (they went below $100 \mathrm{ft}$ above ground level) while executing the stall recovery maneuver without guidance. The load factors are all very similar and well within structural as well as comfort load limits during all runs.

The secondary stall warnings and aerodynamic stall numbers, as shown in Fig. 27(a) reveal similar trends as in the aforementioned low altitude scenario. However, an observable difference in trend with and without 

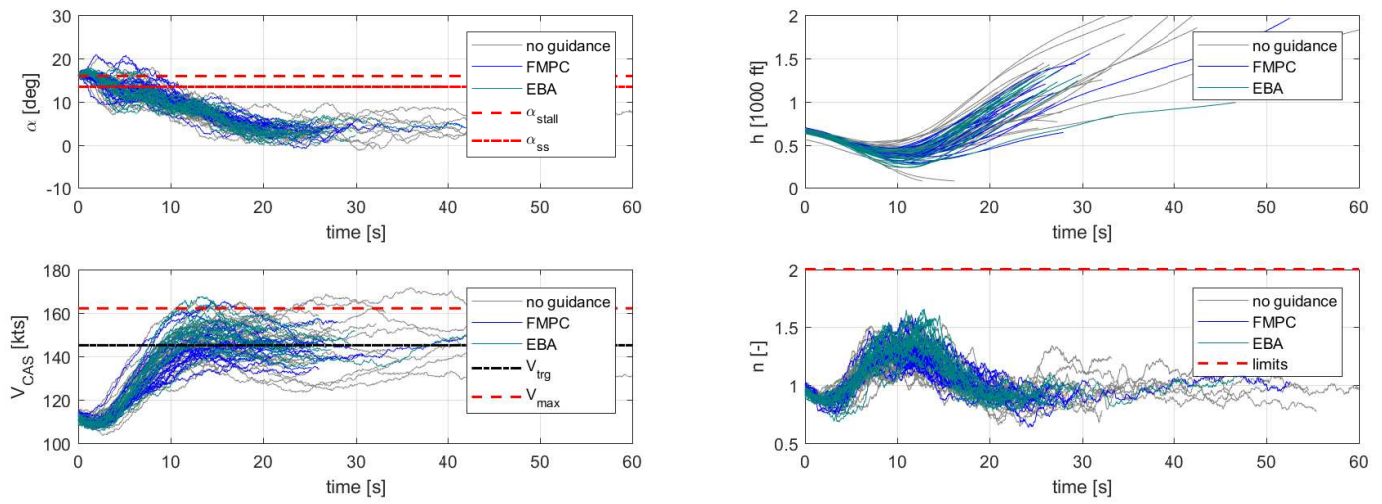

(a) Time histories of angle of attack $\alpha$ and airspeed (b) Time histories of altitude $h$ and load factor $n$ for all $V_{C A S}$ for all runs runs

Figure 26. Time histories of relevant quantities in the approach scenario

guidance is visible in Fig. 27(b), which shows the histogram of the maximum airspeed values during the recovery over all runs and per configuration, and compares them with the target and the overspeed markers. On average, it can be seen that both guidance algorithms resulted in better buffers to overspeed and fewer overspeed exceedances.
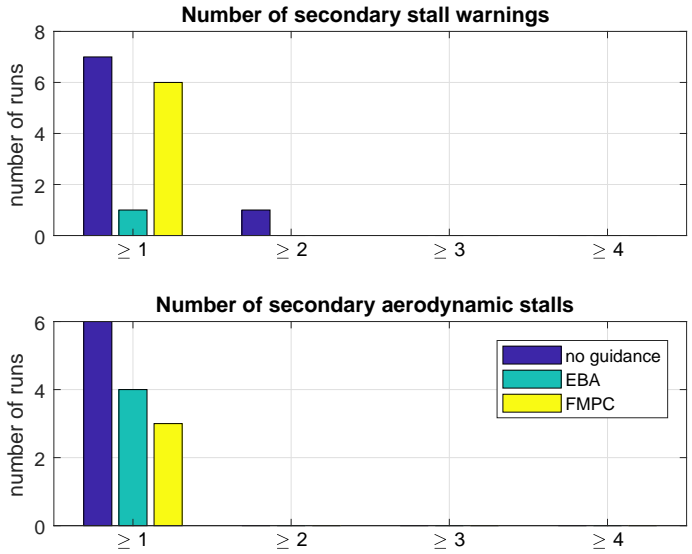

(a) Secondary stick shakers and secondary stalls with and without guidance
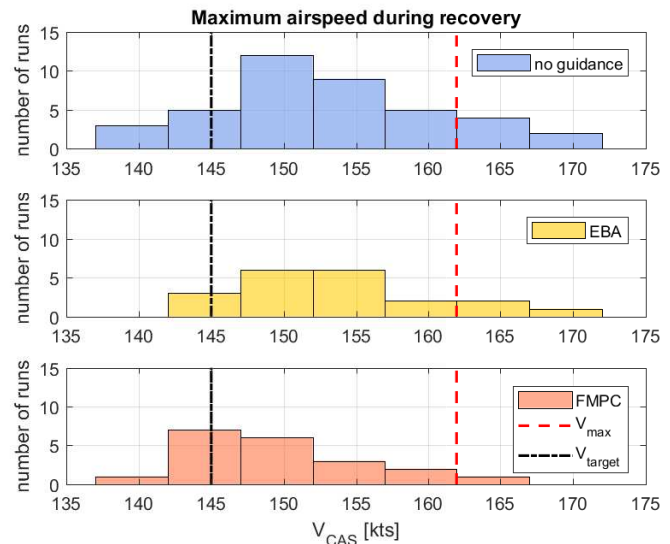

(b) Buffer to maximum flap speed with and without guidance

Figure 27. Impact of guidance on secondary stick shakers and stalls, and overspeed in the approach scenario

\section{E. Pilot Feedback}

Throughout the course of the simulator experiments, pilots completed end-of-run as well as end-of-study questionnaires. Although these questionnaires involved many questions on handling qualities, workload and system usability, this section will focus on the parts about the system usability scale, which was part of the end-of-study survey, and on the handling qualities, which were evaluated in the end-of-run questionnaires. More detailed survey results are discussed in Ref. 24. 


\section{System Usability Scale}

The standard System Usability Scale (SUS) was designed to be a quick and easy survey for a global assessment of a given system's usability ${ }^{34}$ In a SUS survey participants are asked whether or not they agree with 10 statements which are arranged such that positive and negative statements alternate to encourage the respondent to think before responding. A composite score on a scale of $0-100$ (but they are not percentile ranks) is then produced from the responses, with higher scores indicating better usability. The post-simulation questionnaire that each participant was asked to complete after finishing all the simulation runs, included a modified System Usability Scale survey. The survey was modified slightly to make some of the questions more relevant for the evaluation of a stall recovery system, which is typical in the use of SUS surveys for different applications. Figure 28 shows the histogram of the composite SUS rating for the overall guidance system given by the participants. One pilot in the pool gave an outlier rating of 42 . His feedback mentioned confusion about the guidance. Still, the mean usability rating was 81.8 (including the outlier case), which is comparable to the A rating assessment given to other aircraft situation awareness systems studied in Ref. 39

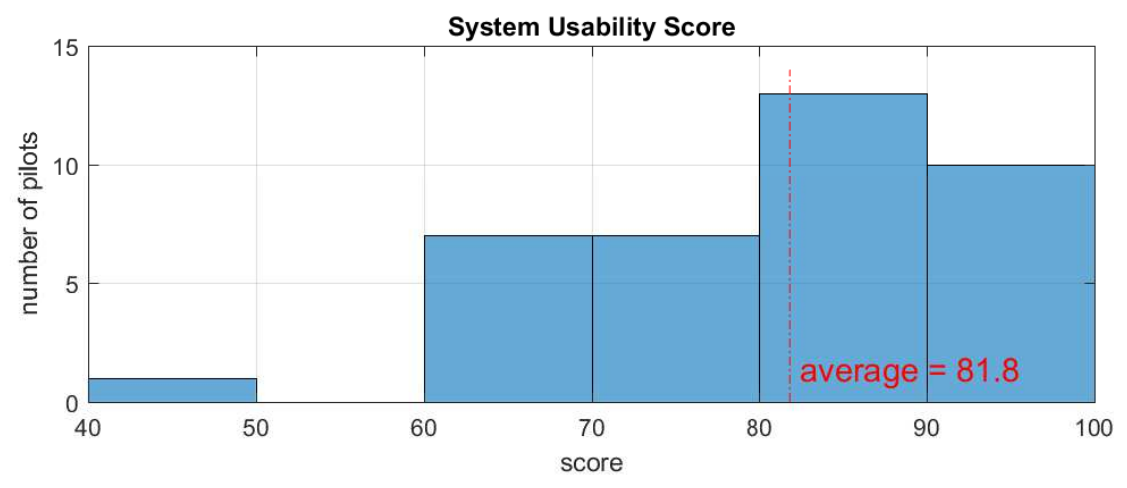

Figure 28. Histogram of the System Usability Scale ratings for the overall guidance system

\section{Cooper Harper Handling Qualities ratings}

Each of the 10 test pilots who participated in the study were asked to provide a Cooper-Harper rating 32$] 33$ (see also the Appendix), on the post run survey immediately after finishing each scenario, with and without guidance. The purpose was to evaluate the impact of the guidance strategies on the handling qualities of the aircraft during the stall recovery. The performance criteria and the relevant values which were used for these evaluations are defined in Tables 5 and 6 . These metrics were presented to the test pilots after finishing each scenario on a separate cockpit display with appropriate color coding, as shown in Fig. 13(b) The Cooper-Harper ratings are presented in Figure 29, grouped per scenario and per algorithm and color coded per pilot. The bulk of the ratings occur between 2 and 6 , indicating a representative result that splits the system performance between the best "satisfactory without improvement" (rating 1-3) category, and the next "deficiencies warrant improvement" (rating 4-6) category. Overall, this result aligned well with the positive subjective feedback provided by most of the participants, along with the constructive suggestions for improving minor aspects of the system. A closer look at Fig. 29 reveals some more details. Fig. 29(a) shows that runs flown by the same pilot with FMPC guidance usually have better handling qualities than without, which also shows in the general spread of ratings. Only for the high altitude scenario, there is no change in the general spread of ratings. It can also be seen that FMPC brings the ratings for the low altitude and approach scenarios within the "satisfactory without improvement" rating category. Ratings for the EBA guidance are presented in Fig. 29(b), Trends here are not as clear as for FMPC. For the high altitude scenario, the aforementioned tendency to lead the pilots into a secondary stall is clear in the ratings with guidance. For the low altitude and approach scenarios, the guidance improved the ratings or kept them 
at least at the same level for more than half of the participants. It should be noted that these observations are based on a small number of ratings, and are not statistically significant. However, the general trends in the ratings confirm the trends that are visible in the tracking performance.

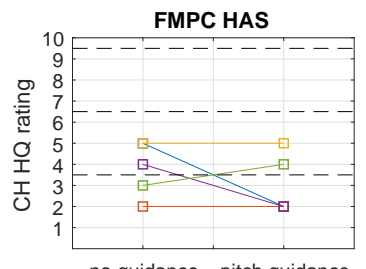

no guidance pitch guidance

FMPC LANUS

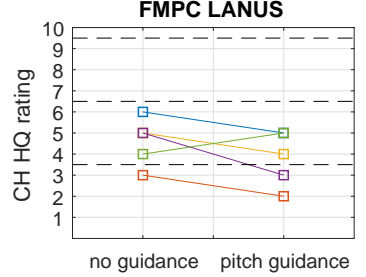

(a) Cooper Harper Ratings for FMPC, all scenarios
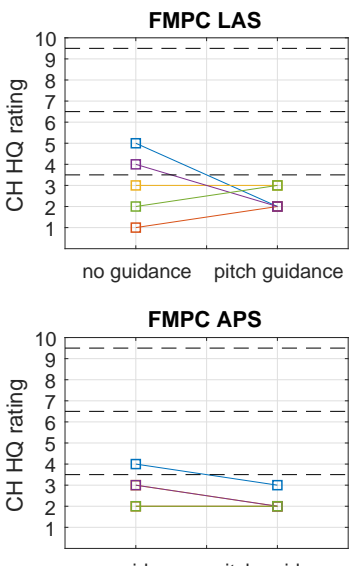

no guidance pitch guidance

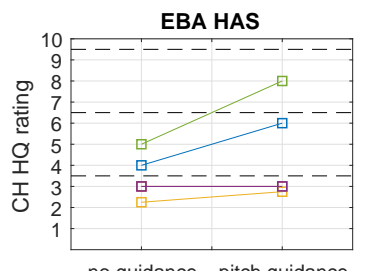

no guidance pitch guidance

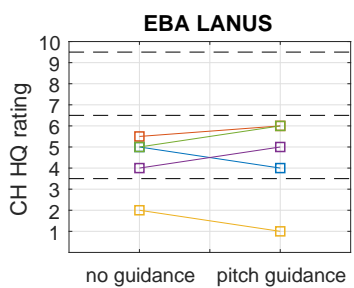

(b) Cooper Harper Ratings for EBA, all scenarios
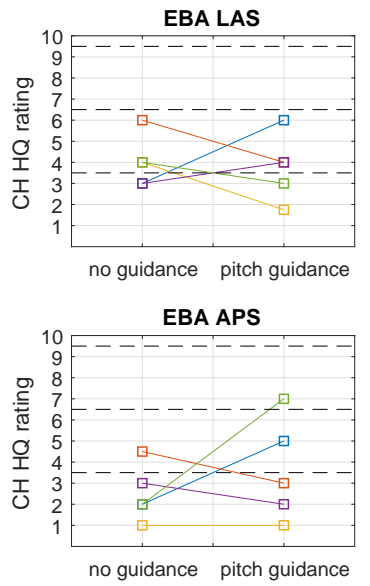

Figure 29. Cooper Harper Handling Quality ratings by test pilots for all scenarios, with and without guidance

\section{F. Minimum Pilot Effort}

Ref. 40 explains that the desire to minimize pilot effort while maximizing the system performance imposes requirements on the dynamic properties of the effective controlled element consisting of the vehicle plus flight director guidance. The human pilot adapts his characteristics to compensate for the dynamic deficiencies of the effective controlled element. As a result of the human pilot properties, a design requirement is that the effective controlled element should be constructed to: (1.) require no low frequency lead equalization and (2.) permit pilot loop closure over a wide range of gains. This can be achieved when the effective controlled element approximates either a pure gain, $\mathrm{K}$, or a pure integration, $\mathrm{K} / \mathrm{s}$, over the frequency range of pilot/director/vehicle system crossover. In short, the key requirement is to adjust the weightings of the various feedbacks in the flight director guidance such that the effective controlled element approximates the $\mathrm{K} / \mathrm{s}$ form over a fairly broad frequency region around the crossover frequency.

Analysis of the block diagram in Fig. 30(a) shows that the 'effective controlled element' as perceived by the pilot during the experiments contains the dynamics between control column and flight director. Fig. 30 (b) shows the frequency response (using a fast fourier transform) of the pitch director displacement to the column input for both algorithms and for all scenarios. It should be noted however that this representation does not consider nonlinear and time varying effects in the dynamics. As a reference, the slope of a single integrator $\mathrm{K} / \mathrm{s}$ is included as well. For most scenarios, the frequency response is very similar to a single integrator. Only for the low frequency range of the high altitude scenario, there is some significant deviant behavior and the frequency response becomes closer to a constant. One can also see that both algorithms behave very similarly. The conclusion of this analysis is that the stall recovery guidance does minimize pilot effort and maximizes system performance.

\section{Conclusions and recommendations}

During this research project, two flight physics based stall recovery guidance algorithms were evaluated in the Vertical Motion Simulator (VMS) at NASA Ames Research Center. One strategy, called fast model predictive control (FMPC), was optimization based. The other, called energy based approach (EBA), was analytical and relied directly on the energy balance between potential and kinetic energy. Performance 


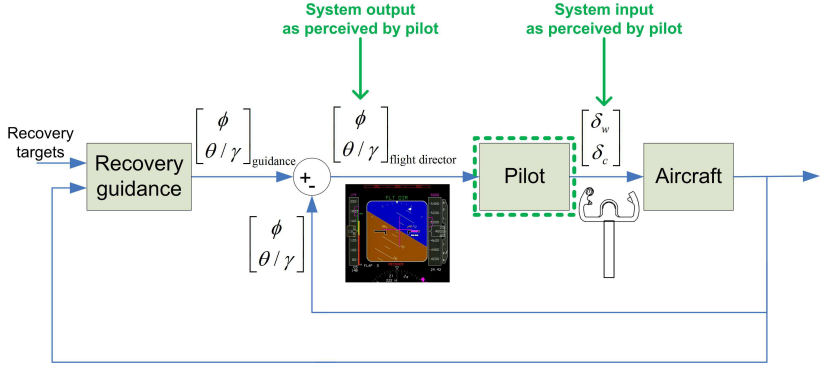

(a) Block diagram illustrating how the airplane behavior is 'perceived' by the pilot

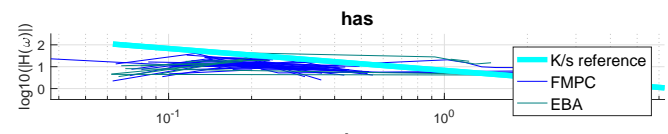

las

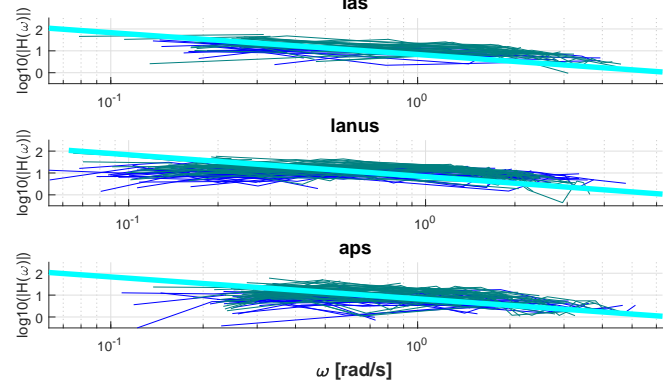

(b) Frequency content from stick to flight director for all scenarios

Figure 30. Analysis of flight director behavior

metrics were compared with and without stall recovery guidance, in order to analyze if the guidance had any beneficial effect for the recovery maneuver. In total, there were 40 participants in this experiment, among them 10 test pilots from Armstrong Flight Research Center. The simulator evaluations considered 4 different scenarios, namely high altitude stall, low altitude stall with and without excessive trim, and approach stall. All these scenarios were inspired by accidents and incidents which occurred over the past years. Both guidance strategies showed similar performance and were well received by the pilots. In the HAS scenario, guidance improved the pilots performance in recovering from stall to the front side of the power curve. Aggressive pitch down guidance in the HAS scenario significantly reduced the tendency for stall warnings and secondary full stall in the recovery. For the low altitude scenario with and without excessive nose up trim incorporating margin to secondary stall warning into the guidance system was beneficial to reducing secondary stall warnings. In the excessive nose up trim scenario, the thrust guidance did help pilots to observe more caution in the application of throttle, and as a result maintained more margin to elevator saturation. In the landing scenario, there was an observable difference in trend that showed better buffers to maximum flap speed and fewer maximum flap speed exceedances with guidance.

It should be noted that no pilot startle or distraction was included in the scenarios. Also, no sensor malfunctions were considered. Both these aspects are crucial for bringing these scenarios closer to real life conditions. It would also be worthwhile to investigate the degree to which longer but shallower dives in the high altitude scenarios could be used for more comfortable recoveries without secondary stalls. Furthermore stall buffet can excite structural modes which can cause issues with handling qualities especially for flexible wing modern aircraft like the Boeing B787. The aeroservoelastic coupling could potentially cause the structural response to feed into the rigid aircraft response which could cause handling qualities issues especially during stall encounters. Finally, stall detection is another important factor to be addressed. All the algorithms discussed here depend on the knowledge where the stall alpha occurs which may not be the case in practical situations. Thus, accurate sensing and detection of stall incipience is necessary.

\section{Acknowledgments}

This work was supported by NASA's Airspace Technology Demonstrations (ATD) project, through the Technologies for Airplane State Awareness (TASA) activity. The authors would like to thank the VMS staff Nick Riccobono and colleagues at NASA Ames Research Center for their support during simulator testing of the developed algorithms. Also a special thanks to Jeff Schroeder and Peter Zaal for their suggestions with respect to the discussion about minimum pilot effort in Sec. $\mathrm{VIF}$ 


\section{Appendix: Cooper Harper Handling Qualities Rating Scale}

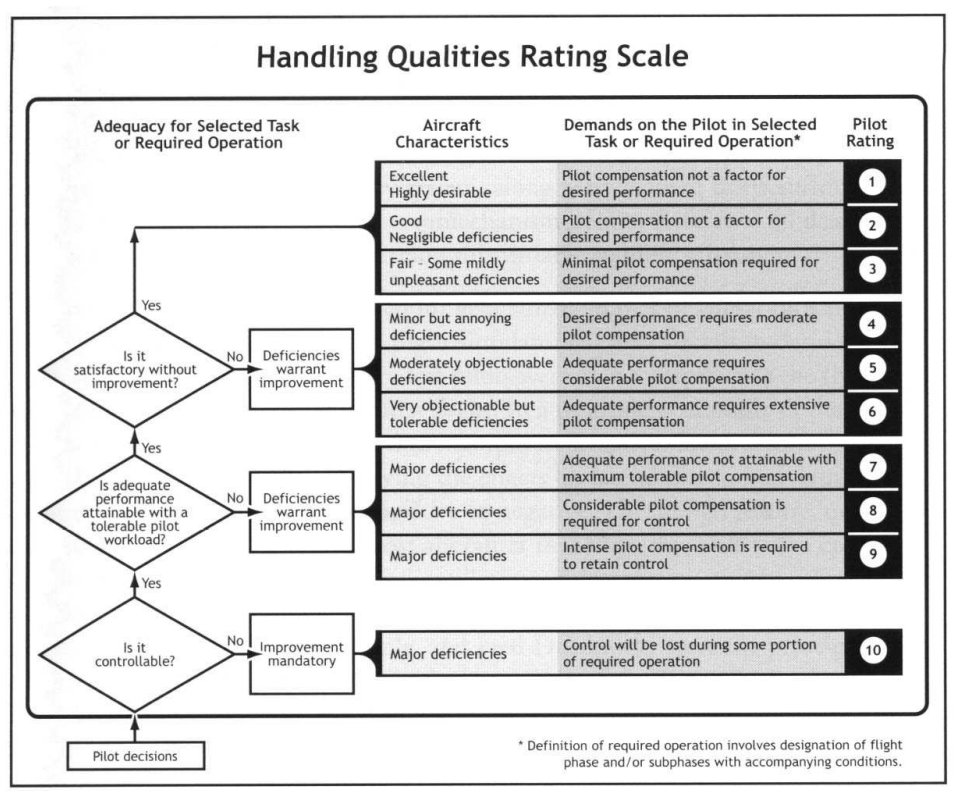

Figure 31. The Cooper Harper Handling Qualities Rating Scale

\section{References}

1 "Global Fatal Accident Review 1980 - 1996," Tech. Rep. CAP 681, CAA - Safety Regulation Group, 1997.

2 "Global Fatal Accident Review 1997 - 2006," Tech. Rep. CAP 776, CAA - Safety Regulation Group, 2008.

3 "Global Fatal Accident Review 2002 - 2011," Tech. Rep. CAP 1036, CAA - Safety Regulation Group, 2013.

4 "Loss of Control on Approach, Colgan Air, Inc., Operating as Continental Connection Flight 3407, Bombardier DHC-8400, N200WQ, Clarence Center, New York, February 12, 2009," Tech. Rep. NTSB/AAR-10/0, National Transportation Safety Board NTSB, Washington, DC, 2010.

5 "Aircraft Accident Investigation Report: PT. Indonesia Air Asia Airbus A320-216; PK-AXC, Karimata Strait, Republic of Indonesia, 28 December 2014," Tech. Rep. KNKT.14.12.29.04, Komite Nasional Keselamatan Transportasi, 2015.

6 "Final Report on the accident on 27 November 2008 off the coast of Canet-Plage to the Airbus A320-232 registered D-AXLA operated by XL Airways Germany," Tech. rep., Bureau dEnqutes et dAnalyses pour la scurit de laviation civile, 2008.

7 "Accident on 24 July 2014 in the region of Gossi in Mali to the MD-83 registered EC-LTV operated by Swiftair S.A." Interim report, Commission d'enquete sur les accidents et incidents d'aviation civile, 2014.

8 "Final Report on the accident on 1st June 2009 to the Airbus A330-203 registered F-GZCP operated by Air France flight AF 447 Rio de Janeiro - Paris," Tech. rep., Bureau dEnqutes et dAnalyses pour la scurit de laviation civile, 2012.

9 "CAST Safety Enhancement Plan: Airplane State Awareness - Effective Upset Prevention and Recovery Training," Technical Report SE196, Commercial Aviation Safety Team, 2013, Available at: www.skybrary.aero/index.php/Portal:CAST_SE_Plan.

10 "CAST Safety Enhancement Plan: Airplane State Awareness - Attitude and Energy State Awareness Technologies," Technical Report SE207, Commercial Aviation Safety Team, 2013, Available at: Www.skybrary.aero/index.php/Portal:CAST_SE_Plan.

${ }^{11}$ Schuet, S., Lombaerts, T., Acosta, D., Wheeler, K., and Kaneshige, J., "An Adaptive Nonlinear Aircraft Maneuvering Envelope Estimation Approach for Online Applications," AIAA SciTech Guidance, Navigation and Control (GNC) Conference, No. AIAA 2014-0268, January 2014, DOI: 10.2514/6.2014-0268.

${ }^{12}$ Shish, K., Kaneshige, J., Acosta, D., Schuet, S., Lombaerts, T., Martin, L., and Madavan, A., "Aircraft Mode and Energy-State Prediction, Assessment, and Alerting," Journal of Guidance, Control and Dynamics, aug 2016, pp. 804 - 816, DOI: 10.2514/1.G001765.

${ }^{13}$ Lombaerts, T., Schuet, S., Acosta, D., Kaneshige, J., Shish, K., and Martin, L., "Piloted Simulator Evaluation of Maneuvering Envelope Information for Flight Crew Awareness," AIAA Guidance, Navigation, and Control Conference, American Institute of Aeronautics and Astronautics (AIAA), jan 2015, DOI: 10.2514/6.2015-1546. 
${ }^{14}$ Lombaerts, T., Looye, G., Ellerbroek, J., and Rodriguez y Martin, M., "Design and Piloted Simulator Evaluation of Adaptive Safe Flight Envelope Protection Algorithm," AIAA Guidance, Navigation and Control Conference, No. AIAA 20160093, January 2016, DOI: 10.2514/6.2016-0093.

15 "Stall Prevention and Recovery Training," Advisory Circular 120-109A, US Department of Transportation, Federal Aviation Administration, November 2015.

${ }^{16}$ Belcastro, C., "Loss of Control Prevention and Recovery: Onboard Guidance, Control, and Systems Technologies," AIAA Guidance, Navigation, and Control Conference, 2012, DOI: 10.2514/6.2012-4762.

${ }^{17}$ Gandhi, N., Richards, N. D., and Bateman, A. J., "Desktop Simulator Demonstration of a Joint Human/Automated Upset Recovery System," AIAA Guidance, Navigation and Control Conference, No. AIAA-2012-4820, 2012.

${ }^{18}$ Richards, N. D., Gandhi, N., and Bateman, A. J., "Improved Upset Recovery Strategies through explicit Consideration of Pilot Dynamic Behavior," AIAA Guidance, Navigation and Control Conference, No. AIAA-2012-4821, 2012, DOI: $10.2514 / 6.2012-4821$.

${ }^{19}$ Gandhi, N., Richards, N. D., and Bateman, A. J., "Simulator Evaluation of an In-Cockpit Cueing System for Upset Recovery," AIAA SciTech, No. AIAA-2014-0444, 2014, DOI: 10.2514/6.2014-0444.

${ }^{20}$ Richards, N. D., Gandhi, N., Bateman, A. J., Klyde, D., and Lampton, A., "Development and Pilot-In-The-Loop Evaluation of Robust Upset-Recovery Guidance," AIAA SciTech, No. AIAA-2016-0879, 2016, DOI: 10.2514/6.2016-0879.

${ }^{21}$ Stepanyan, V., Krishnakumar, K., Kaneshige, J., and Acosta, D., "Stall Recovery Guidance Algorithms Based on Constrained Control Approaches," AIAA SciTech, No. AIAA-2016-0878, 2016, DOI: 10.2514/6.2016-0878.

${ }^{22}$ Lombaerts, T., Schuet, S., Kaneshige, J., Shish, K., and Stepanyan, V., "Stall Recovery Guidance Using an Energy Based Algorithm," AIAA SciTech Conference, No. AIAA-2017-1021, American Institute for Aeronautics and Astronautics, 2017.

${ }^{23}$ Schuet, S., Lombaerts, T., Kaneshige, J., Shish, K., and Stepanyan, V., "Stall Recovery Guidance Using Fast Model Predictive Control," AIAA SciTech Conference, No. AIAA-2017-1513, American Institute for Aeronautics and Astronautics, 2017.

${ }^{24}$ Schuet, S., Lombaerts, T., Stepanyan, V., Kaneshige, J., Shish, K., Robinson, P., and Hardy, G., "Vertical Motion Simulator Experiment on Stall Recovery Guidance," Tech. Rep. NASA/TP-2017-219733, NASA, January 2017.

${ }^{25}$ Hueschen, R. M., "Development of the Transport Class Model (TCM) Aircraft Simulation From a Sub-Scale Generic Transport Model (GTM) Simulation," Technical Report NASA/TM2011-217169, NASA Langley Research Center, Hampton, Virginia, August 2011, Available at: http://ntrs.nasa.gov/archive/nasa/casi.ntrs.nasa.gov/20110014509.pdf.

${ }^{26}$ Campbell, S., Kaneshige, J., Nguyen, N., and Krishnakumar, K., "An Adaptive Control Simulation Study using Pilot Handling Qualities Evaluations," AIAA Guidance, Navigation, and Control Conference, No. AIAA-2010-8013, 2010.

${ }^{27}$ Hyde, D. C., Brown, F., Shweyk, K. M., and Shah, G., "Advanced Modeling and Uncertainty Quantification for Flight Dynamics; Interim Results and Challenges," Proceedings of the AIAA SciTech Conference, No. AIAA-2014-0035, 2014, DOI 10.2514/6.2014-0035.

${ }^{28}$ Wang, Y. and Boyd, S., "Fast model predictive control using online optimization," Control Systems Technology, IEEE Transactions on, Vol. 18, No. 2, March 2010, pp. 267-278.

${ }^{29}$ Rutowski, E., "Energy Approach to the General Aircraft Performance Problem," Journal of the Aeronautical Sciences, Vol. 21, No. 3, March 1954, pp. 187-195, DOI: 10.2514/8.2956.

${ }^{30}$ Lambregts, A., "Functional integration of vertical flight path and speed control using energy principles," First Annual NASA Aircraft Controls Workshop, NASA Langley Research Center, 1983, https://ntrs.nasa.gov/search.jsp?R=19840012520.

${ }^{31}$ Merkt, J., "Flight Energy Management Training: Promoting Safety and Efficiency," Journal of Aviation Technology and Engineering, Vol. 3, No. 1, 2013, pp. 24 36, DOI: 10.7771/2159-6670.1072.

${ }^{32}$ Cooper, G. and Harper, R., "The use of pilot rating in the evaluation of aircraft handling qualities," Technical Note TN D-5153, National Aeronautics and Space Administration, Washington D.C., April 1969, URL: http://www.dtic.mil/get-trdoc/pdf?AD =AD0689722.

${ }^{33}$ Harper, R. and Cooper, G., "Handling qualities and pilot evaluation," Journal of Guidance, Control and Dynamics, Vol. 9, No. 5, September-October 1986, pp. 515-529, DOI: 10.2514/3.20142.

${ }^{34}$ Brooke, J., Usability Evaluation in Industry, chap. SUS - A quick and dirty usability scale, Taylor \& Francis, 1986.

${ }^{35}$ Danek, G., "Vertical motion simulator familiarization guide," Technical report NASA-TM-103923, NASA Ames Research Center, May 1993, URL: https://ntrs.nasa.gov/search.jsp?R=19940008535.

${ }^{36}$ Beard, S., Reardon, S., Tobias, E., and Aponso, B., "Simulation System Optimization for Rotorcraft Research on the Vertical Motion Simulator Read More: https://arc.aiaa.org/doi/abs/10.2514/6.2012-4634," AIAA Modeling and Simulation Technologies Conference, No. AIAA 2012-4634, American Institute of Aeronautics and Astronautics, 2012.

${ }^{37}$ Lewis, E. and Vuong, N., "Integration of Matlab Simulink Models with the Vertical Motion Simulator," AIAA Modeling and Simulation Technologies Conference, No. AIAA-2012-4797, American Institute of Aeronautics and Astronautics, 2012.

38 "Descent Below Visual Glidepath and Impact With Seawall, Asiana Airlines Flight 214, Boeing 777-200ER, HL7742, San Francisco," Tech. Rep. NTSB/AAR-14/01, National Transportation Safety Board NTSB, Washington, DC, 2014.

${ }^{39}$ Evans, E., Young, S., Daniels, T., Santiago-Espada, Y., and Etherington, T., "Analysis of pilot feedback regarding the use of state awareness technologies during complex situations." IEEE/AIAA 35th Digital Avionics Systems Conference (DASC), IEEE, September 2016, pp. 1 - 9, DOI: 10.1109/DASC.2016.7777995.

${ }^{40}$ Weir, D., Klein, R., and McRuer, D., "Principles for the design of advanced flight director systems based on the theory of manual control displays," contractor report NASA CR-1748, prepared by Systems Technology, Inc. for NASA Ames Research Center, Hawthorne, CA, March 1971. 